\title{
Analysis of Users and Uses of DEMs in Spain
}

\author{
José Luis Mesa-Mingorance ${ }^{1, *}$ (1) , Elena Gabriela Chicaiza ${ }^{2}$, Xavier Buenaño ${ }^{2}$, Jianhong Cai ${ }^{3}$, \\ Antonio Federico Rodríguez-Pascual ${ }^{4}$ and Francisco Javier Ariza-López ${ }^{1, *}$ \\ 1 Departamento de Ingeniería Cartográfica, Geodésica y Fotogrametría, Universidad de Jaén, 23071 Jaén, Spain \\ 2 Departamento de Ingeniería Geográfica, Universidad Politécnica de Madrid, 28031 Madrid, Spain; \\ gabriela.chicaizam@alumnos.upm.es (E.G.C.); xavier.buenanog@alumnos.upm.es (X.B.) \\ 3 School of Surveying and Mapping Engineering, Beijing University of Civil Engineering and Architecture, \\ Beijing 100044, China; cjh@bucea.edu.cn \\ 4 Centro Nacional de Información Geográfica, Instituto Geográfico Nacional, 28003 Madrid, Spain; \\ afrodriguez@fomento.es \\ * Correspondence: jlmesa@ujaen.es (J.L.M.-M.); fjariza@ujaen.es (F.J.A.-L.); \\ Tel.: +34-953-212-849 (J.L.M.-M.); +34-953-212-469 (F.J.A.-L.)
}

Received: 24 October 2017; Accepted: 15 December 2017; Published: 20 December 2017

\begin{abstract}
We have carried out a characterization of users and uses of Digital Elevation Models (DEMs), in order to be able to study an adaptation of the most appropriate DEM. In the previous literature, there have not been many similar studies of this subject. We used information about DEMs downloaded from a Download Center of a National Mapping Service (in this case the Centro Nacional de Información Geográfica, CNIG, of Spain). This service offers three DEM products with different spatial resolutions (DEM05, DEM25 and DEM200). We employed a total of 12,493 records from an online survey. The completion of the survey was mandatory at the time of the download (year 2014). We determined the geographical location of downloads, the profile of users, the use of the DEMs and the user assessment. We identified 6087 different users, most with a profile of private professionals $(71 \%)$ and related educational activities (18\%). Most of the users performed only one download. The major uses are those related to teaching-research and professional activities. Uses related to leisure, sport and tourism were $9.5 \%$ of all cases. The valuation performed by users of the utility of the products was very high, but not particularly in relation to updating needs.
\end{abstract}

Keywords: DEM; use; user

\section{Introduction}

There is an abundance of digital geospatial data products in most countries, and this is especially true in Spain (e.g., orthophotos, elevation models, street maps, etc.). The existence of this abundant geographic information has been achieved thanks to the work performed by many data producers from the government at different levels (national, regional, local), from the private sector (e.g., Google, Microsoft, etc.), and even by the citizenship (e.g., volunteered geographic information). In the case of Spain, the majority of data providers belong to the public administration and they are many, e.g., one National Mapping Agency (Instituto Geográfico Nacional, IGN), several thematic institutes (Geological, Marine, etc.), several ministries (e.g., Agriculture, Public Finances), and many regional cartographic agencies (IECA, IGC, etc.), all of them coordinated by the High Geographic Council (Consejo Superior Geográfico), and several cartographic laws [1,2].

The abundance and access of geospatial data have grown in parallel, and both have undergone a major transformation in the last decade [3]; from costly and sometimes complicated access to easy access, high transparency and availability. There are metadata and view access online without any costs, download systems, etc. In the case of Europe, these achievements have been possible, in some 
degree, thanks to the legal framework established by the European Union, principally by the Inspire Directive [4]. The INSPIRE directive aims to create a European Union spatial data infrastructure for the purposes of EU environmental policies and policies or activities which may have an impact on the environment. This European Spatial Data Infrastructure will enable the sharing of environmental spatial information among public sector organizations, facilitate public access to spatial information across Europe and assist in policy-making across boundaries. (https://inspire.ec.europa.eu/about-inspire/563).

In Spain, as in other countries, much attention has been paid to the creation of data products, to implementing geoservices, mainly Web Map Services (WMS), and to creating the corresponding metadata and catalogue services; and even to analyzing the economic benefits of investing in spatial data infrastructure (e.g., [5]). However, from the point of view of the authors there are not enough studies regarding the uses and users of spatial data products. All industrial production, whether of goods or general services, is based solely on the value and utility that generates its use [6], and we think the same applies to geomatics products. Total Quality [7] and other Quality Management System proposals (e.g., ISO 9001 [ISO 2015], as well as the European Foundation for Quality Management (EFQM) model [8], etc.) are using Deming's Circle [9], where user feedback is an important issue, in order to develop a continuous improvement strategy. In this line, the document NTCA-02 (Normas Técnicas Cartográficas de Andalucía) [10], a standard of the Cartographic and Statistics Institute of Andalusia (IECA) based on Deming's Circle, is a clear example in the geomatics sector: it establishes the principle that spatial-data users' opinions and satisfaction must be surveyed periodically. Therefore, we consider this study as novel because it allows us to know in more detail the users and uses to which DEMs are principally assigned. We have found no articles that address this topic in depth. We believe that knowing the user and main use of a DEM can positively influence the productive process, the approach, design, capture of information and finally representation or final expression of DEMs.

Digital Elevation Models (DEM) are a key data type for many applications domains. DEMs provide the height component in GIS analysis, the geomorphological description of the land [11], a reference surface for all hydrological applications (water cycle, erosion, floods, etc.) [12], the basis for the development of forestry models [13], the base for agricultural parcel rating [14] and are useful in every analysis task related to civil engineering [15].

In Europe, DEMs are considered baseline data and belong to the list of datasets of the Annex II of INSPIRE (Infrastructure for spatial information in Europe). In the case of Spain, the transposition of INSPIRE is reflected in the law (Law 14/2010) [16], and this indicates that DEMs are national reference data. According to the legislation of the European Union these data must be accessible to all citizens and administrations. This is achieved by mapping services and download services. In Spain DEMs are freely downloadable and usable provided that their use is not commercial (http:/ / centrodedescargas. cnig.es/CentroDescargas/cambiarMenu.do?destino=infoUsoNoComercial). This facilitates the spread of knowledge and its use.

The aim of this paper is to perform an accurate and current description of users and uses of DEMs in Spain. Our major goals are: (i) to obtain knowledge of users' profiles, and (ii) to learn in what kind of applications DEMs are being used. This study is of interest and would have relevance in justifying investments, segmenting the market, and identifying trends and potential opportunities for the development of new value-added products. We have been able to analyze the situation in Spain because we have had access to data coming from the Download Center (http: / / centrodedescargas. cnig.es/CentroDescargas/index.jsp), the download service from the Centro Nacional de Información Geografica (CNIG), the commercial branch of the IGN. This online download service contains a survey which must be answered by the user. The survey answering was mandatory until the end of 2014, but nowadays is voluntary. This paper presents a statistical analysis based on counts, percentages and crossing of answers given by the DEM-data downloaders. 


\section{Materials and Methods}

The bases for this analysis are the answers provided by DEM-data downloaders from the Download Center of the CNIG for the year 2014 (January to December). Figure 1 presents the online-survey form offered to downloaders. To facilitate the survey analysis, and in order to obtain standardized responses, some items allow the selection of a single option from an enumerated list, and other items a multiple selection on a checklist. There are also free text items (Descriptive) for the introduction of comments. Table 1 shows the items and data types presented in Figure 1.

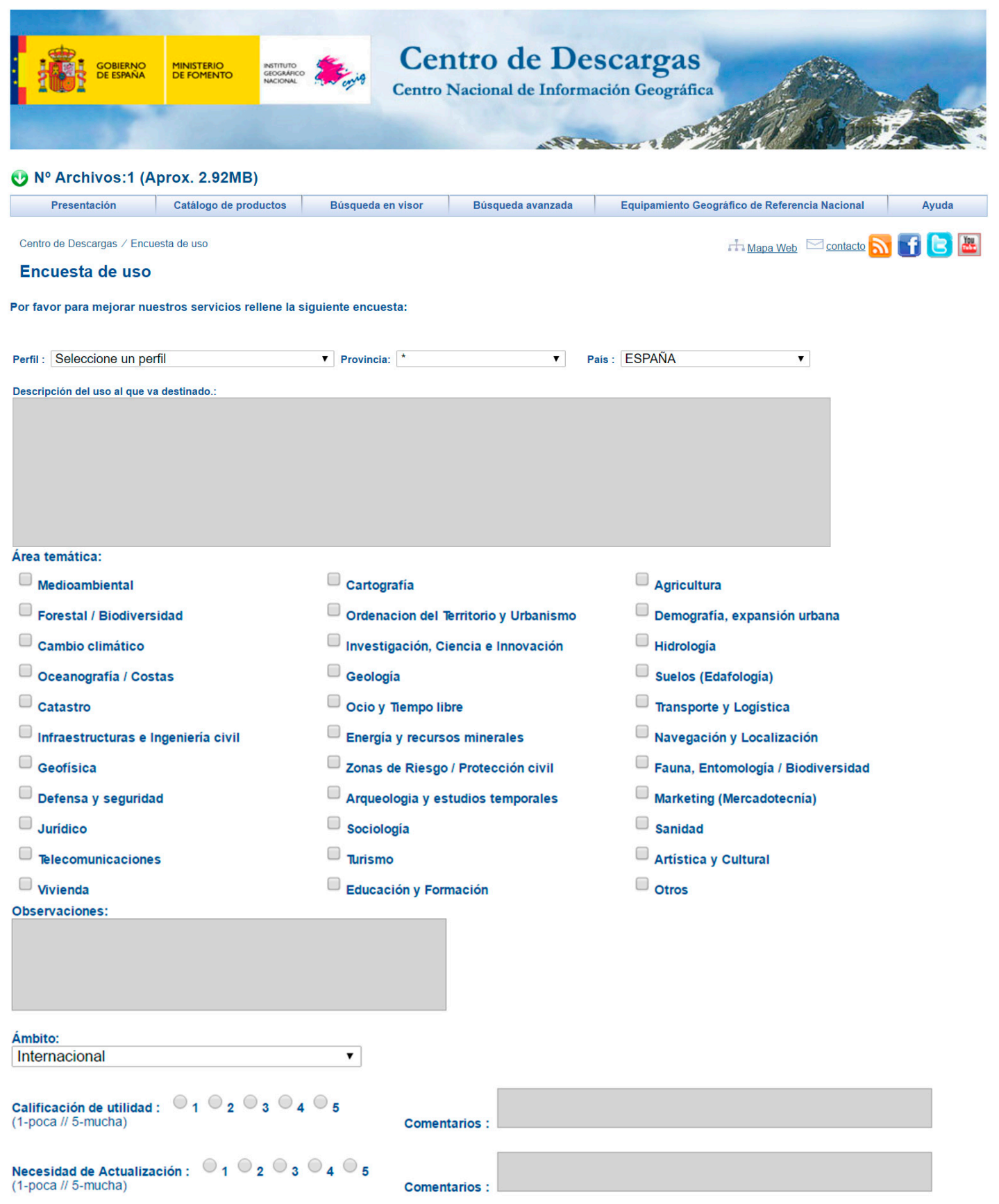

Figure 1. Web survey template of the Download Center (CNIG). 
Table 1. Items of the web survey template of the Download Center (CNIG).

\begin{tabular}{|c|c|}
\hline Item & Possible Values \\
\hline \multirow{3}{*}{ DEM } & DEM05 \\
\hline & DEM25 \\
\hline & DEM200 \\
\hline Date & Date of download \\
\hline \multirow{10}{*}{ User profile } & Particular use \\
\hline & Microenterprise (<10 employees) \\
\hline & Small and Medium enterprises (<50 employees) \\
\hline & Medium enterprises (between 51 to 250 employees) \\
\hline & Large company (>250 employees) \\
\hline & General State Administration \\
\hline & Regional Government \\
\hline & University \\
\hline & Local Government \\
\hline & Others \\
\hline \multirow{5}{*}{ Utility } & 1 unhepful \\
\hline & 2 \\
\hline & 3 \\
\hline & 4 \\
\hline & 5 very useful \\
\hline Comments on the utility & Descriptive (Free text) \\
\hline \multirow{5}{*}{ Need to update } & 1 little need \\
\hline & 2 \\
\hline & 3 \\
\hline & 4 \\
\hline & 5 much need \\
\hline Comments on the need to update & Descriptive (Free text) \\
\hline Province & Province (52) \\
\hline Country & Country \\
\hline Description of the intended use & Descriptive (Free text) \\
\hline \multirow{20}{*}{$\begin{array}{c}\text { Thematic Area (employment sectors DEM) } \\
\text { (In this item you can select several } \\
\text { thematic areas) }\end{array}$} & Environmental \\
\hline & Forest/Biodiversity \\
\hline & Climate Change \\
\hline & Oceanography/Costs \\
\hline & Cadaster \\
\hline & Infrastructure and Civil Engineering \\
\hline & Geophysics \\
\hline & Defense and Security \\
\hline & Legal \\
\hline & Telecommunications \\
\hline & Living Place \\
\hline & Cartography \\
\hline & Planning and Urbanism \\
\hline & Research, Science and Innovation \\
\hline & Geology \\
\hline & Leisure and free time \\
\hline & Energy and mineral resources \\
\hline & Risk areas/Civil protection \\
\hline & Archaeology and temporary studies \\
\hline & Sociology \\
\hline
\end{tabular}


Table 1. Cont.

\begin{tabular}{cc}
\hline Item & Possible Values \\
\hline & Tourism \\
& Education and formation \\
Agriculture & \\
& Demographic and urban expansion \\
Hydrology & Soils (soil science) \\
& Transport and logistics \\
& Navigation and location \\
& Wildlife, Entomology /Biodiversity \\
& Marketing \\
& Health \\
& Artistic and cultural \\
& Others \\
\hline Comments on the sectors & Descriptive (Free text) \\
& International \\
& Europe \\
Area & National \\
& Regional \\
& Province \\
& Other (Local, watershed, etc.) \\
\hline
\end{tabular}

It is important to note that there are 3 different DEMs available for downloading. All of them are distributed among files following the same distribution of sheets of the Spanish base map at scale $1 / 25,000$. The Coordinates follow the official system (ETRS89 + UTM projection) and the file format is ESRI (asc). The differences come from sources and resolution:

- DEM05. A DEM grid with $5 \times 5 \mathrm{~m}$ resolution. For the production of this DEM two data sources have been used: (i) automatic stereo-correlation from photogrammetric flights of the Ortophoto National Plan (ground sample distance $=25 \mathrm{~cm}$ and $50 \mathrm{~cm}$ ), revised and interpolated with break lines where feasible, (ii) LIDAR flights with 0.5 point $/ \mathrm{m}^{2}$.

- DEM25. A DEM grid with $25 \times 25 \mathrm{~m}$ resolution. This product was obtained from the previous one by generalization.

- DEM200. A DEM grid with $200 \times 200 \mathrm{~m}$ resolution. This product was obtained from the first one by generalization. It is distributed by province not by sheets.

We had at our disposal a total of $\mathrm{N}=12,493$ records from users who completed the survey for all DEM downloads performed throughout 2014. First, we proceeded to analyze how to set up a process in order to be able to use all data in a later statistical study (cleaning process). As mentioned above, there are selection fields and multiple-choice fields (e.g., Thematic Area), but also free-text response items. Descriptive items present a great variety of answers (e.g., words, sentences, long texts, etc.); a situation that makes any quantitative computation difficult. Therefore, regarding statistical analysis, the major problem comes from this kind of item, and a normalization process of the responses will be needed in order to enable further analysis. Next, we will describe the processes of data preparation.

We normalized all items that allow free text entries. These items are: "Comments about the sectors", "Description of intended use", "Comments on the utility". We worked with all of them because these titles suggest the possibility of interesting information given by the users. First we have to indicate that not all records contain entries for these items. In relation to the total $\mathrm{N}$ (12,493 records), the percentage of items completed is $4.64 \%$ with respect to the item "Comments about the sectors", $99.95 \%$ with respect to the item "Description of intended use" and 1.53\% with respect to the item "Comments on the utility". The normalization process was performed manually, a tedious and time-consuming task. Normalization was performed jointly for all the records of the three products being analyzed, 
in order to be homogeneous. The normalization process consisted of replacing the contents of these items (e.g., words, sentences, phrases or long texts) with a single term (key term) in order to summarize and classify the answer. We have proposed 44 key terms to standardize the answers and they are presented in Table 2. The consideration of each one of these key terms reflects the idea of collecting, as closely as possible, all existing cases without loss of generality. The generation of this list of key terms was an iterative process: the progress of analysis of the records induced the entrance/exit, aggregation/disaggregation, change of meaning/scope of all the terms until that moment of the analysis.

Table 2. Uses: standard terms.

\footnotetext{
Standard Terms

1. Acoustic. There are DEM applications in the field of noise analysis (e.g., near airports, cities, etc.).

2. Agriculture. All activities related to agriculture are included.

3. GIS analysis. Processes include slope calculating, slope orientation, viewshed, data transformation, etc.

4. Archaeological Heritage. All activities related to archeology and heritage are included.

5. Architecture. All activities related to architecture. Spatial planning is under another term.

6. Aviation. All activities related to aviation (flights, approaches, etc.) are included.

7. Biology. All activities related to plants, insects, etc., which have a more specific description than those listed under the generic term of an environmental nature are included.

8. Cadastre Market. All activities related to land, plots and valuation and market aspects (e.g., geomarketing) are included. The number of records regarding land exceeds the market.

9. Testing. Numerous records indicate "test", "test values", "Checking altitudes", etc. All these cases are included under this term. It really is a non-specified case but it is interesting that the sense of this term is given verification. 10. Curiosity. Numerous records indicate that users approach these products out of curiosity. It is understood that they perform specific applications, simply making contact with the product, usually in a geographic and thematic
} environment which would be known.

11. Security Defense Civil Protection. All activities related to defense, security and civil protection services, leaving aside fires for their specificity.

12. Teaching Study. Under this term all the activities related to teaching, at all levels including university and short training courses are included. They are listed as simple teaching and class preparation by teachers (theoretical or practical). Records related to the study, i.e., activities performed by students at all levels, are also included.

13. Renewable Energy. All activities related to solar energy (thermal and photovoltaic), biomass, etc. are included.

14. Forest. All activities related to the mountains and forest species are included.

15. Geography Territory. All activities related to knowledge of the geographical environment and territory are included. Planning and action on the territory is considered under another term.

16. Geology. All activities related to geology, geodynamics, soil science, etc. are included.

17. Geomatic. All activities related to mapping, map generation, DEM management, applications in Geodesy,

Photogrammetry and Remote Sensing applications, Survey applications, etc. are included.

18. Hydrology. All activities related to surface and coastal water are included.

19. History. All activities related to history are included.

20. Environmental Impact. All activities related to studies and environmental impact projects are included. They are considered outside the general term of Environment for its specificity.

21. Fires. All activities related to fire are included.

22. Civil Engineering. All activities related to any activity of civil engineering are included, excluding agriculture, forestry and telecommunications.

23. Research. All records in which this term appears but does not specify the field of research are included.

Teaching and Research have been standardized as Research, as well as doctoral theses.

24. Legal Judicial Administrative Tender. All records relating to legal, judicial, administrative or tender preparation activities are included.

25. Location. Many users use this term to describe how they use the DEM.

26. Environment. A broad category in which all activities related to the environment and that have not been considered under another heading are included.

27. Mining. All records related to mining activities are included.

28. Modeling. Many users use this term to describe how they use the DEM. Within this category hydrological modeling (Hydrology) and 3D (3D visualization) modeling are not included.

29. Navigation. All records related to navigation are included.

30. Leisure Sports Tourism. All records related to sport and leisure activities (hiking, trekking, bike routes, etc.) and tourism are included.

31. Landscape. All records related to landscape are included. 
Table 2. Cont.

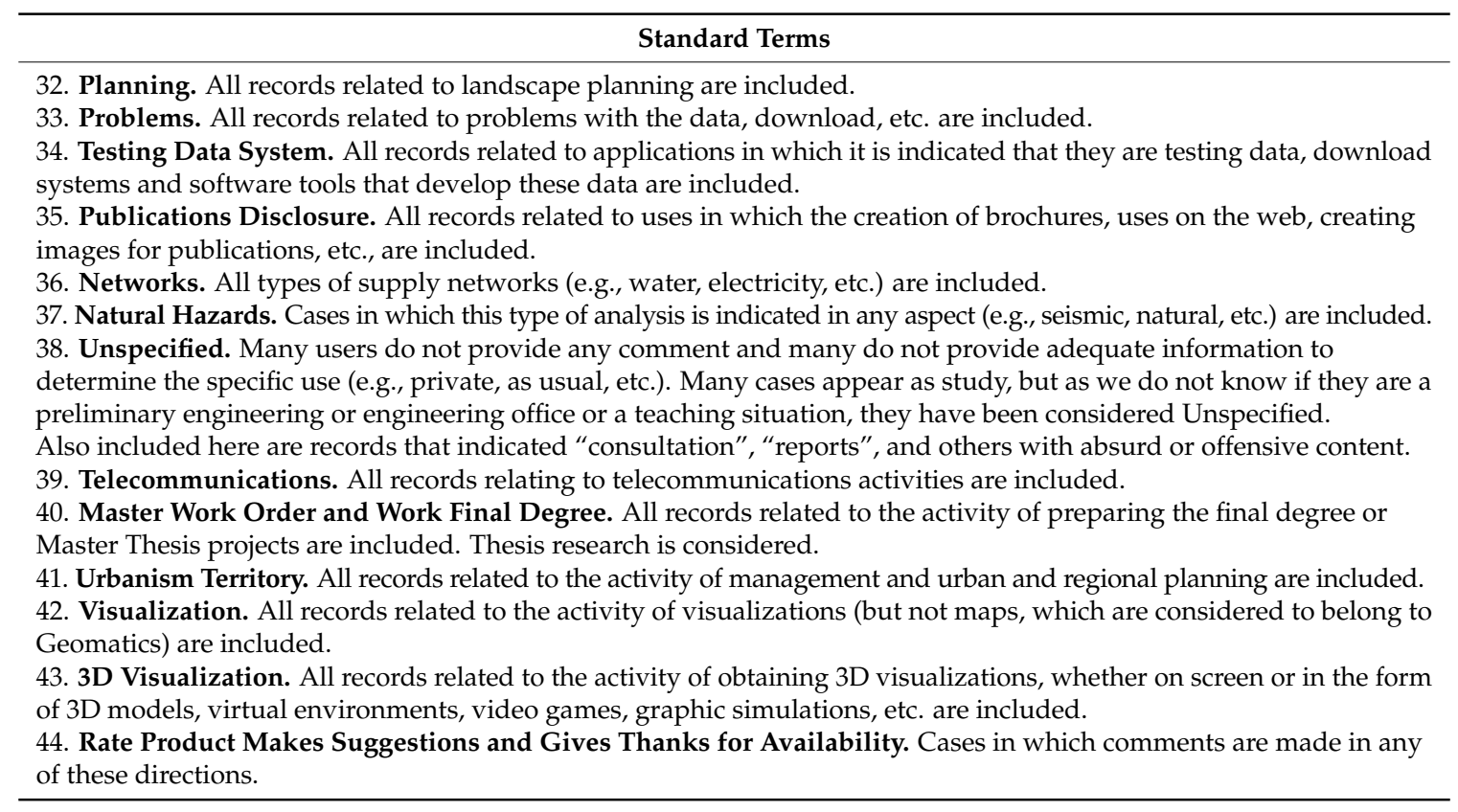

The item "Thematic Area" (Sectors) may include a number of different responses (multiple options). In order to properly address this item we have converted it into a set of Boolean variables where each one takes value 1 when the user has set that option for a given sector.

Finally, regarding the methods for the data analysis, standard tools of the descriptive statistics were used (e.g., means, proportions, cross tabulation techniques, etc.).

\section{Data Analysis, Results and Discussion}

The results of our analysis are presented and discussed in this section. They are presented in the following subsections:

- Location and product downloading.

- User profile.

- Use of the product.

- Valuation of the product.

\subsection{Location and Product Downloading}

We will present the results concerning the download frequency of each product and the location indicated by the users. Regarding downloads by product; results are shown in Table 3 where the highest percentage of downloads corresponds to the DEM05. It can be seen that the amount of downloads decreases when increasing the grid size (lower spatial resolution). The reason for this may be the users' tendency to use the product with the better performance, in this case with the better spatial resolution, regardless of whether or not it is the most suitable for a specific task.

Table 3. Data concerning the type of DEM download.

\begin{tabular}{ccc}
\hline DEM & Number of Records & \% of Total \\
\hline DEM200 & 1377 & 11.02 \\
DEM25 & 4180 & 33.46 \\
DEM05 & 6936 & 55.52 \\
\hline
\end{tabular}


In relation to the location of the download, the survey has several items to determine the place where users reside, and where the download occurs. The majority of downloads take place in Spain $(12,470)$, and downloads in other countries are minimal. France, Portugal and the United Kingdom account for 3 or 4 downloads. It is interesting to note that a long list of Hispano-American countries appear with only one download. We think that the use of the same language makes it easy to test this download system.

Figure 2 presents two maps with the distribution of downloads in Spain by provinces. Figure $2 \mathrm{a}$ represents the percentage of the total downloads in each province. Figure $2 \mathrm{~b}$ represents the number of downloads per thousand per province and relative to its population (official population data published by the Instituto Nacional de Estadística for 2014). In the first map we used the method of Natural Break Intervals for its representation. In this case data are grouped creating intervals so that the internal variance of the intervals is less than the variance between intervals. This method is similar to a statistical cluster and we obtain the groups that are presented in the population. For the second map we used intervals of equal amplitude in order to represent a variable which behaves very regularly. In Figure 2a it can be seen that the province of Madrid holds the highest percentage of downloads (approximately 18\%), and this is a very noticeable difference from the other provinces. At first it appears that this high percentage of downloads in Madrid could be due to its high population and economic importance, although in this respect we should also highlight other provinces such as Barcelona. However, in the case of Barcelona there is a regional mapping service (Instituto Cartográfico $y$ Geológico de Cataluña, ICGC) that provides an alternative downloading system for this region of Spain. In the second interval $([2.81,4.39])$, there are seven provinces of peripheral character (La Coruña, Barcelona, Valencia, Alicante, Murcia, Granada, Seville) and an interior province (Zaragoza). This is interesting because coastal provinces in Spain are more dynamic and usually have more economic power than interior provinces. Finally, in this Figure we highlight the existence of an east-west strip of land consisting of provinces where the lowest percentage of downloads was detected.

The percentage of downloads in each province was represented in Figure $2 \mathrm{a}$ and can be detailed a little more if we take into account the population of each province. Figure $2 \mathrm{~b}$ represents per-thousand downloads for the total population in each province. Thus, it complements the perspective of Figure 2a. Figure $2 b$ includes provinces such as Avila, Cuenca and Albacete in the range of higher values. In the case of Avila, we believe this may be due to the existence of a university with studies related to Geomatics; and as we will see later, the university world is a big user of DEMs. In this case the periphery effect also exists, but with a different sense to that of Figure 2a. The relation between downloads and population is not linear: downloads increase with population but not proportionally, so that the ratio per-thousands of downloads decreases with the increase of population. It is important to notice the low level of this ratio in the regions of Cataluña and Valencia. We believe this is motivated by regional mapping agencies which allow the downloading of similar products. 


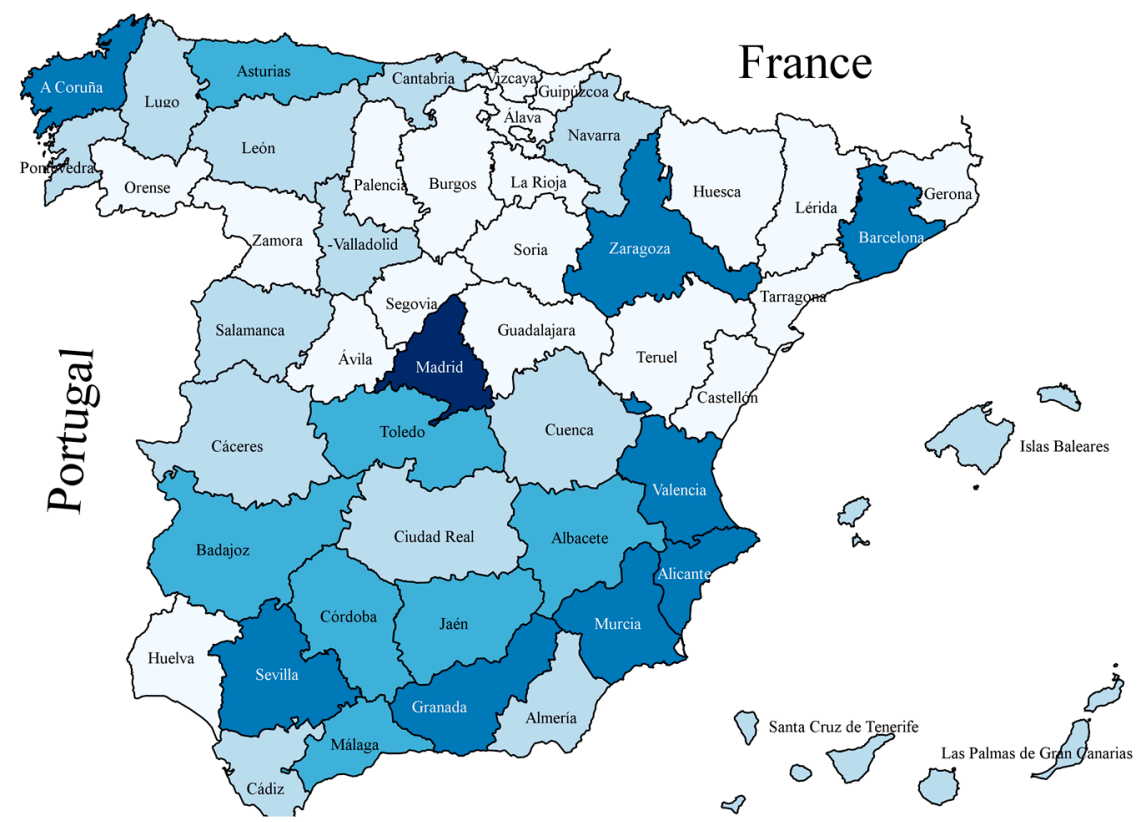

Percent downloads to total downloads

Provinces

$\square[0.43-1.02]$
$\square(1.02-1.84]$
$\square(1.84-2.81]$
$\square(2.81-4.39]$
$\square(4.39-18.45]$

(a)

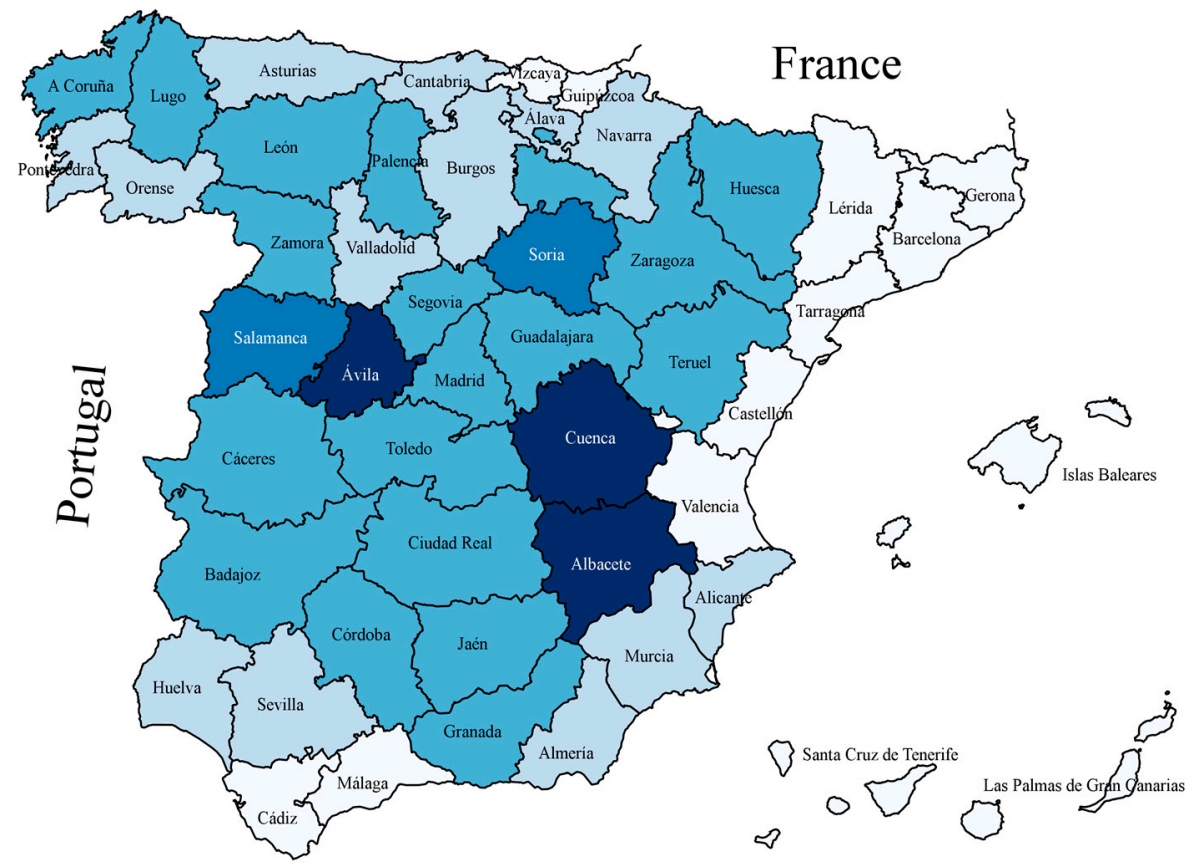

Downloads regarding the population of the province (per thousand)

Provinces
\begin{tabular}{|l}
$\square[0.07-0.21]$ \\
$\square(0.21-0.35]$ \\
$\square(0.35-0.48]$ \\
$\square(0.48-0.62]$ \\
$\square(0.62-0.76]$
\end{tabular}

(b)

Figure 2. Distribution by province of downloads in Spain: (a) percentage of the total downloads, (b) number of downloads per thousand inhabitants (relates downloads with the population of each province). 


\subsection{User Profile}

One of our main objectives is to establish users' profiles. In the download system, a user is each person or institution that is registered in the system and has a different identification. Thus, several people can access under the same identity, and one person can access with different identifications. With these limitations, we were able to identify a total of 6087 different users in the period considered. This was possible because we had a unique identifier provided by the IGN (Instituto Geográfico Nacional) as a combination of name and e-mail of the downloader. The survey form has 9 options that can determine user's profiles of various kinds, whose analysis is straightforward and is shown in Table 4. The profile with more weight is that related to particular users, where freelances are included. The second most important user profile corresponds to the universities, including teaching, research and final degree preparation or similar work. As expected, smaller companies have a higher percentage of downloads than larger ones. The public administrations (national, regional and local) accumulate $3.99 \%$ of total downloads. All of this indicates that the predominant use $(>95 \%)$ is not related to the work of public administrations. Considering each of the three downloadable products, Table 4 shows that there is a great similarity in the download behavior. In relation to the spatial resolution (grid size), it is interesting to note that for administrations and enterprises the percentage of downloads increases with the increase of resolution. For the case of universities and private users, the situation is the opposite. We think that public administrations and enterprises work at greater scales (local) and that universities work with smaller scales (regional or national).

Table 4. Downloads by user profile.

\begin{tabular}{ccccc}
\hline User Profile & \% All Products & \% DEM200 & \% DEM25 & \% DEM05 \\
\hline General State Administration & 1.79 & 1.53 & 1.10 & 2.26 \\
Local Administration & 0.97 & 0.44 & 0.81 & 1.17 \\
Regional Administration & 1.23 & 0.87 & 0.81 & 1.56 \\
Large company (>250 employees) & 0.82 & 0.29 & 0.77 & 0.97 \\
Medium-sized business (between 51 and 250 employees) & 0.74 & 0.73 & 0.50 & 0.89 \\
Small business (<50 employees) & 1.16 & 0.94 & 0.89 & 1.37 \\
Micro business (<10 employees) & 3.39 & 1.45 & 2.61 & 4.25 \\
University & 17.71 & 20.26 & 18.54 & 16.70 \\
Private use & 70.74 & 71.90 & 72.42 & 69.51 \\
Others & 1.43 & 1.60 & 1.56 & 1.33 \\
Total & 100.00 & 100.00 & 100.00 & 100.00 \\
\hline
\end{tabular}

If we cross the profile of user marked in the survey and the standard comments of use, we observe that there are some inconsistencies. For all user profiles the standard comment on the majority use is Teaching-Study. In some profiles, such as medium-sized businesses, the second most frequent comment is Leisure-Sports-Tourism, which does not seem logical. In third and fourth position of the number of downloads appear standard comments of the most coherent use, thus in Regional Administration, Large Company and Medium-sized business Geomatics appears in third place and in some other profiles research appears in fourth place, as in the profile University. These last standard comments on the use of DEMs seem more logical. However, with the most standard comment use being related to Teaching-Study it makes us think that regardless of the user's profile downloading for the development of work related to Geomatic, Environmental, Geological, Biological studies, etc. are the ultimate goals of those downloaded DEMs.

Figure 3 shows the relation between user profiles and sectors. For clarity purposes, for each user profile only the four sectors accounting for the largest numbers of cases are represented, and dots are drawn in a log-scale. As can be observed, only ten sectors have representation in this figure (rows with dots), and two of them are predominant (Agriculture and Research, Science and Innovation). The last is of interest because all user profiles perform applications in these two sectors. Leaving aside the case of "others", Education and Training and Archeology and Temporal Studies are also highlighted in this figure. We can highlight the relation between Archeology and temporal studies with Local and 
Regional Governments and Microenterprises. In Spain, these two administrations (Local and Regional) are responsible for the historical heritage, and free professionals (microenterprises) usually perform these tasks. Finally, the importance of Education and Training is obvious for the University user profile but Figure 3 also indicates that DEMs are used in particular uses for training (self-training?).

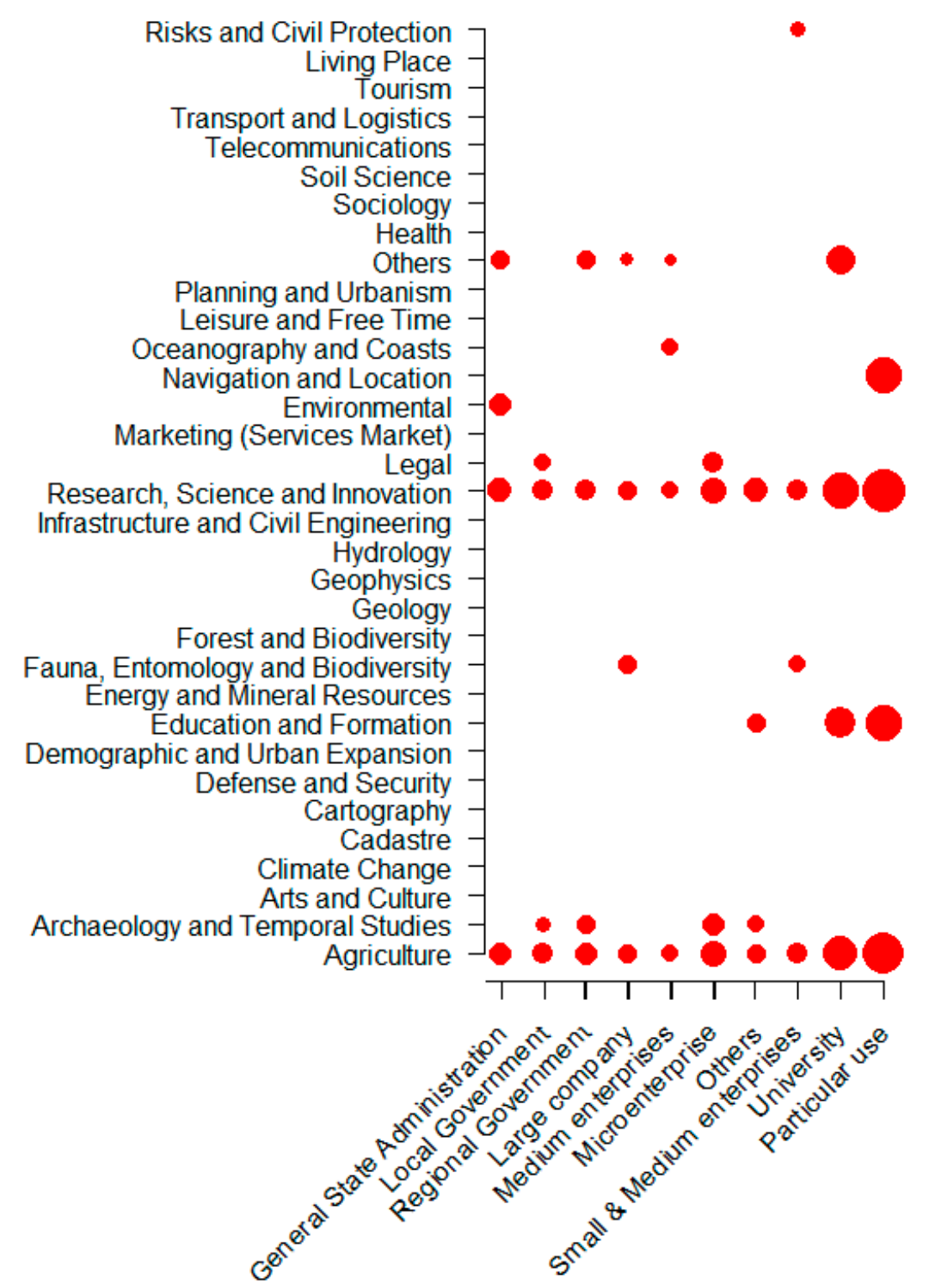

Figure 3. The relation between user profile and sectors (thematic area).

Next, we can establish the relationship between the user profile and the level of utility that they give to the DEM. Figure 4 shows the percentage of each level of utility granted by each user profile.

As shown in Figure 4, utility is well valued by all the profiles, with level 5 always receiving more than $50 \%$, especially in large companies where the percentage is the highest of all in level 5 of utility. However, where the percentage of level 5 is lower is in the profile of medium-sized businesses and regional administration. It is difficult to obtain justified conclusions that explain this behavior, since there are no obvious reasons. However, it can be observed in the downloads percentages of Table 4 that both profiles have percentages of DEM200 downloads very close to DEM05 values, that is to say that both low resolution and high-resolution models are used in both profiles. This may be because 
the objective of the work covers a large area of territory. Probably working on a large area of territory is in studies where the utility of the DEM loses importance with respect to other cartographic models.

On the other hand, we have analyzed the behavior of the percentage of the levels assigned to the need to update the DEM in each user profile (Figure 5).

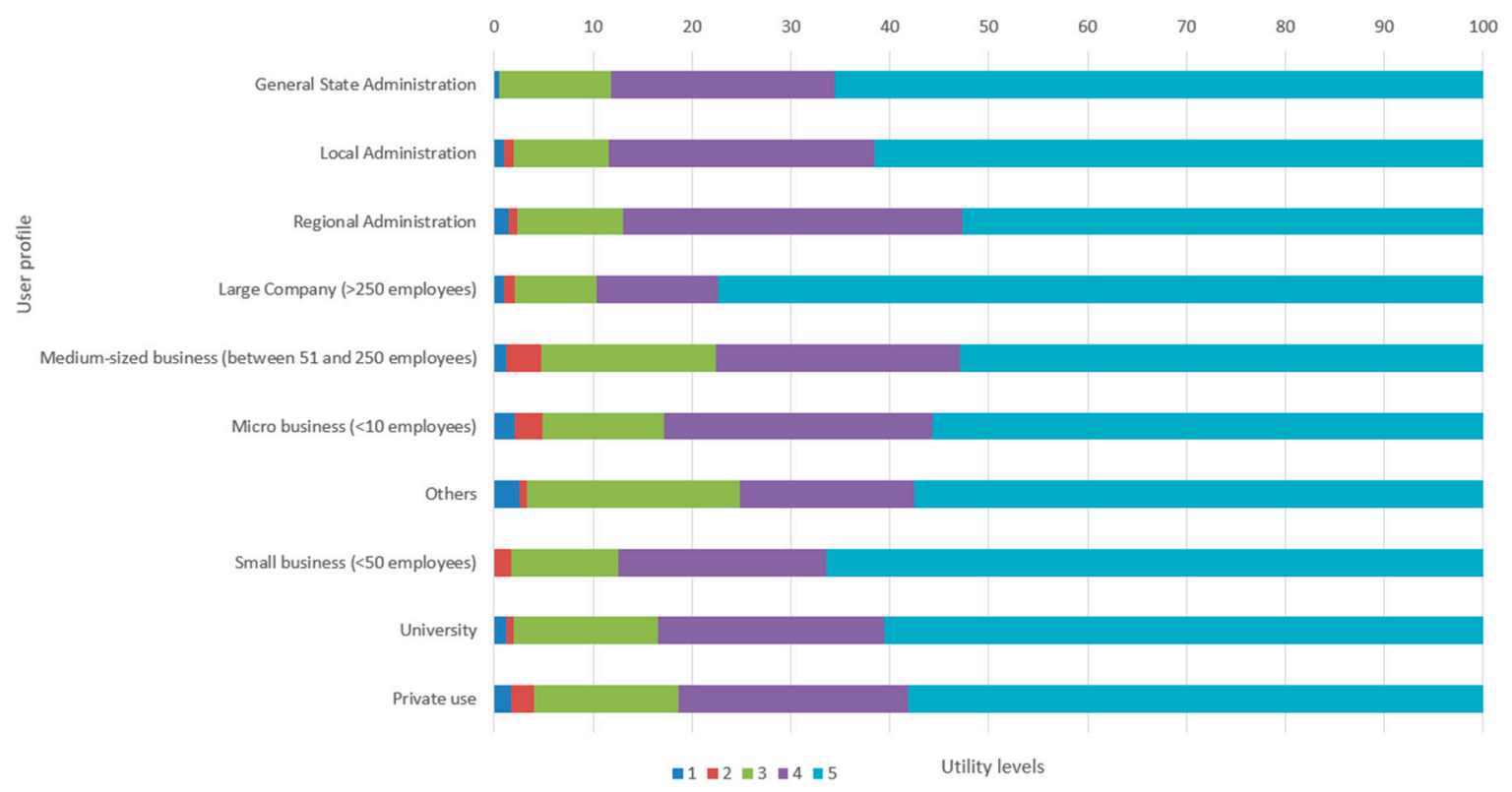

Figure 4. Percentage of the utility levels assigned per user profile.

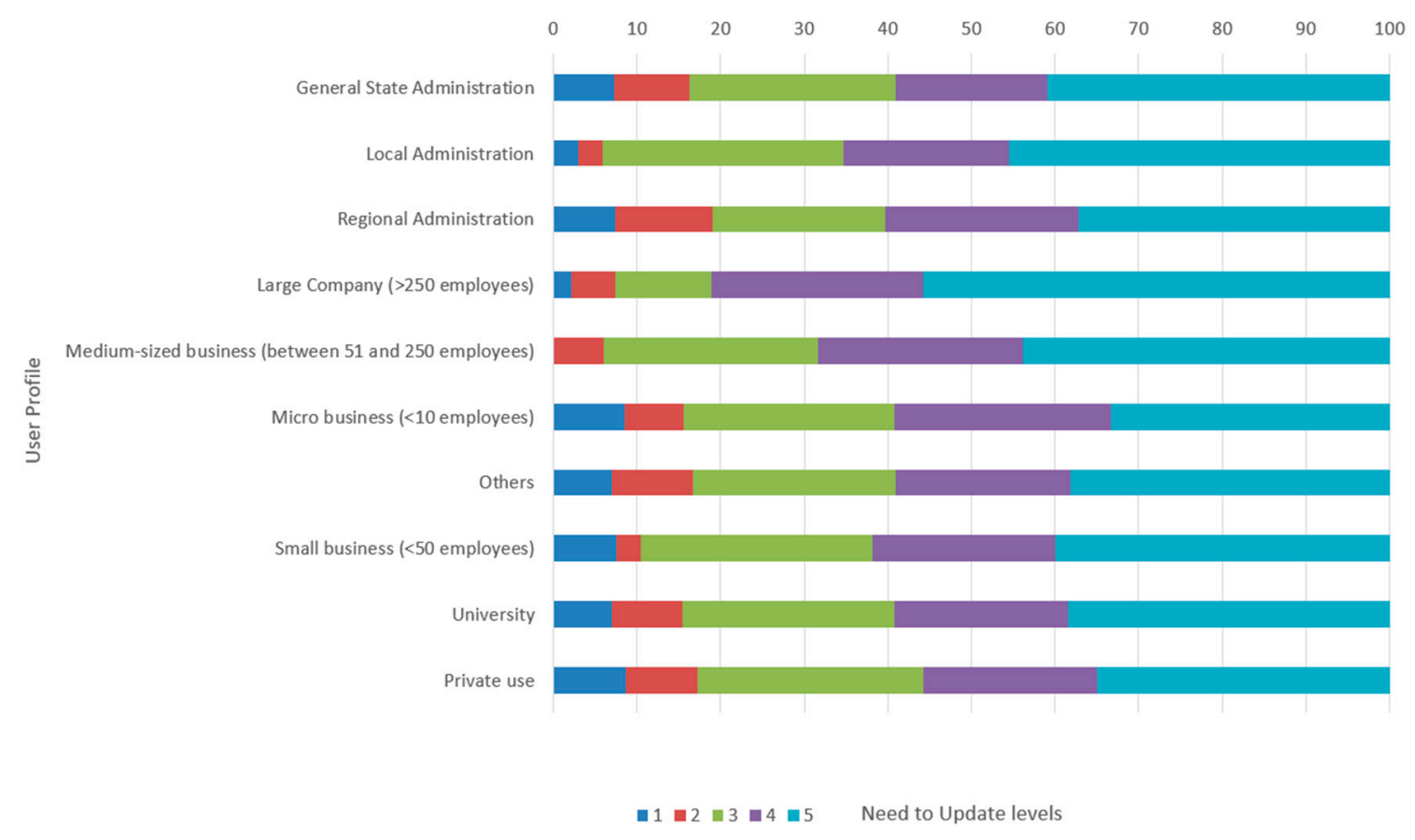

Figure 5. Percentage of the need to update levels assigned per user profile.

In this case the percentages of level 5 are lower, not exceeding $50 \%$ in any user profile, except in large companies, which as with utility is the user profile with the highest percentage of level 5 assigned. The profile of large companies has the most marked thematic area: Environmental, Cartography and Infrastructure and Civil Engineering, being the only profile that includes Infrastructures and Civil Engineering among the most marked. This could be one of the reasons why both utility and the need 
to update at level 5 are considered to be higher, since infrastructures need a shorter update period due to their constant change, and therefore they need a more detailed and updated knowledge of the environment. Even so, the need to update is an aspect less valued by the user than the utility of the DEM.

Other interesting information is that related to the registration profile, and Table 5 presents the available data. Here the more important issue is that the majority of users are recorded as people, independently of whether they work for a company / administration or not.

Table 5. Downloads by individual or company.

\begin{tabular}{ccc}
\hline Profile & $\mathbf{N}$ & $\mathbf{\%}$ \\
\hline Company & 64 & 1.0 \\
Individual Person & 5897 & 96.7 \\
No answer/don't know & 136 & 2.2 \\
Total & 6097 & 100.0 \\
\hline
\end{tabular}

Another aspect of interest is the activity performed by users, that is the number of downloads performed in the year of analysis. Figure 6 shows the number of users (vertical axis) against the number of downloads (horizontal axis). As can be seen, the number of downloads ranges from 1 to 39 , but the vast majority of users only perform one download. The most common number of downloads is between 1 and 3 . Users who perform 1 or 2 downloads accumulate $80 \%$ of cases, and users who perform up to 5 downloads accumulate $95 \%$ of cases.

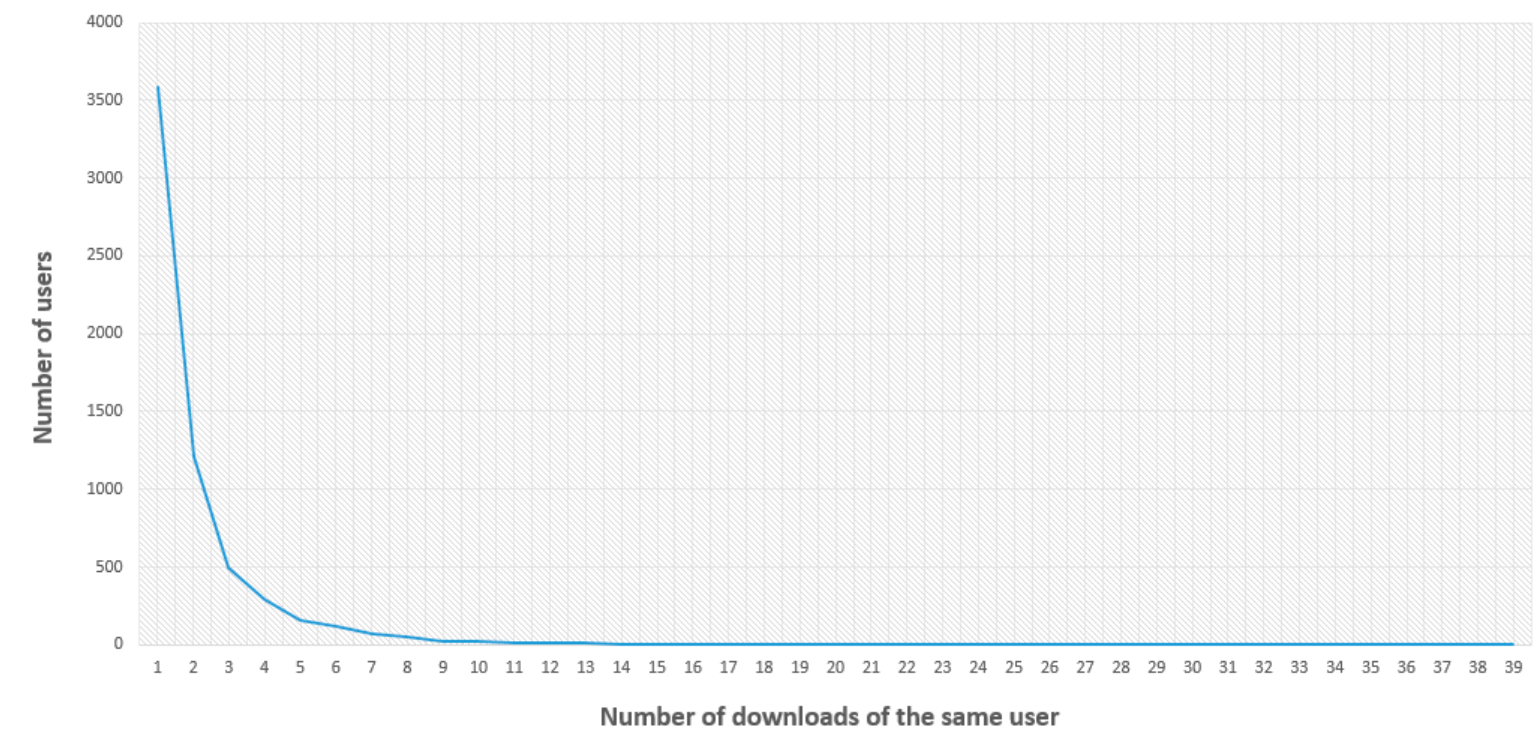

Figure 6. Number of downloads performed by the same user.

In view of the results we might think that most users are occasional users, as they perform 1 or 2 downloads. However, we must clarify this statement based on download user habits, the download system and the survey system. The survey system includes a record for each download, but the download can include multiple files. Indicating different download habits, for example file by file or several files together, produces a number of different records although the number of files downloaded may finally be the same.

\subsection{Use of the Product}

To characterize the use of the product, we can use the item "Thematic Area" (Sectors) from the survey form. This item provides the ability to mark a multiple choice in order to indicate all the sectors 
where they will use the products. In addition, in order to complement this information, the user is offered an item for comments ("Comments about the sectors"), and a free text item ("Description of intended use"). The item "Description of intended use" was normalized with the terms of Table 2. Here we present the results from these items.

Table 6 shows the frequency with which they have selected one or more options in relation to these Sectors or Thematic Areas. Nearly 73\% of users mark a single thematic area. We consider that this indicates a strong focus on specific implementations. Almost $95 \%$ of users are included in the group of the first three categories of the table, that is, they mark 3, 2 or 1 in the item Thematic Area. There are cases of users who indicate a large number of areas, in some cases even selecting all options. However, the percentage of these cases is very low.

Table 6. Number of users according to the number of thematic areas selected in each download.

\begin{tabular}{cccc}
\hline Number of Areas Marked & Users & Number of Areas Marked & Users \\
\hline 1 & 9130 & 18 & 2 \\
2 & 1878 & 19 & 4 \\
3 & 758 & 20 & 0 \\
4 & 286 & 21 & 1 \\
5 & 159 & 22 & 4 \\
6 & 86 & 23 & 0 \\
7 & 43 & 24 & 0 \\
8 & 44 & 25 & 1 \\
9 & 26 & 26 & 0 \\
10 & 23 & 27 & 0 \\
11 & 11 & 28 & 0 \\
12 & 8 & 29 & 0 \\
13 & 7 & 30 & 0 \\
14 & 5 & 31 & 0 \\
15 & 7 & 32 & 2 \\
16 & 2 & 33 & 6 \\
17 & 0 & & \\
\hline
\end{tabular}

Table 7 presents the distribution of the accounts received by each Thematic Area. The figure included in the table represents the $\%$ of downloads with respect to the total number of downloads; and the figures the percentages in relation to the total number of records. This information clearly indicates the main Thematic Areas of use for the DEMs in Spain. In this way applications related to Cartography and the Environment are clearly more numerous, with more than 3000 counts $(60.57 \%)$. A second group of activities are those that are in the vicinity of 1000 counts $(42.62 \%)$, which are: Hydrology, Education and Formation, Leisure and Free Time, Planning and Urbanism, Forest and Biodiversity. We could establish a third group around the value of 500 counts $(54.69 \%)$ and a last one below this value.

In order to obtain a graphical representation of "Thematic areas" of use, a network graphic (Figure 7) was derived using a force-directed graph algorithm (in this case the igraph library in $\mathrm{R})$. This graph presents one node for each thematic area, and their size is proportional to the number of checks of each thematic area. The edge widths have been plotted in a color scale range according to the weights of relations, that is, the connections between thematic areas have a different thickness depending on the times that the user jointly indicates both thematic areas, but only the most representative relations are shown. The strongest relation occurs between Cartography and the Environmental sectors, and both have a strong relation with Hydrology and Forest. There is also a remarkable relation between Urban and Regional Planning, Cartography and Geology. There are sectors such as Health, Telecommunications, Marketing, Legal and Sociology which do not show highlighted relations. 
Table 7. Percentage of downloads in each of the Thematic Areas. (Sorted by percentage).

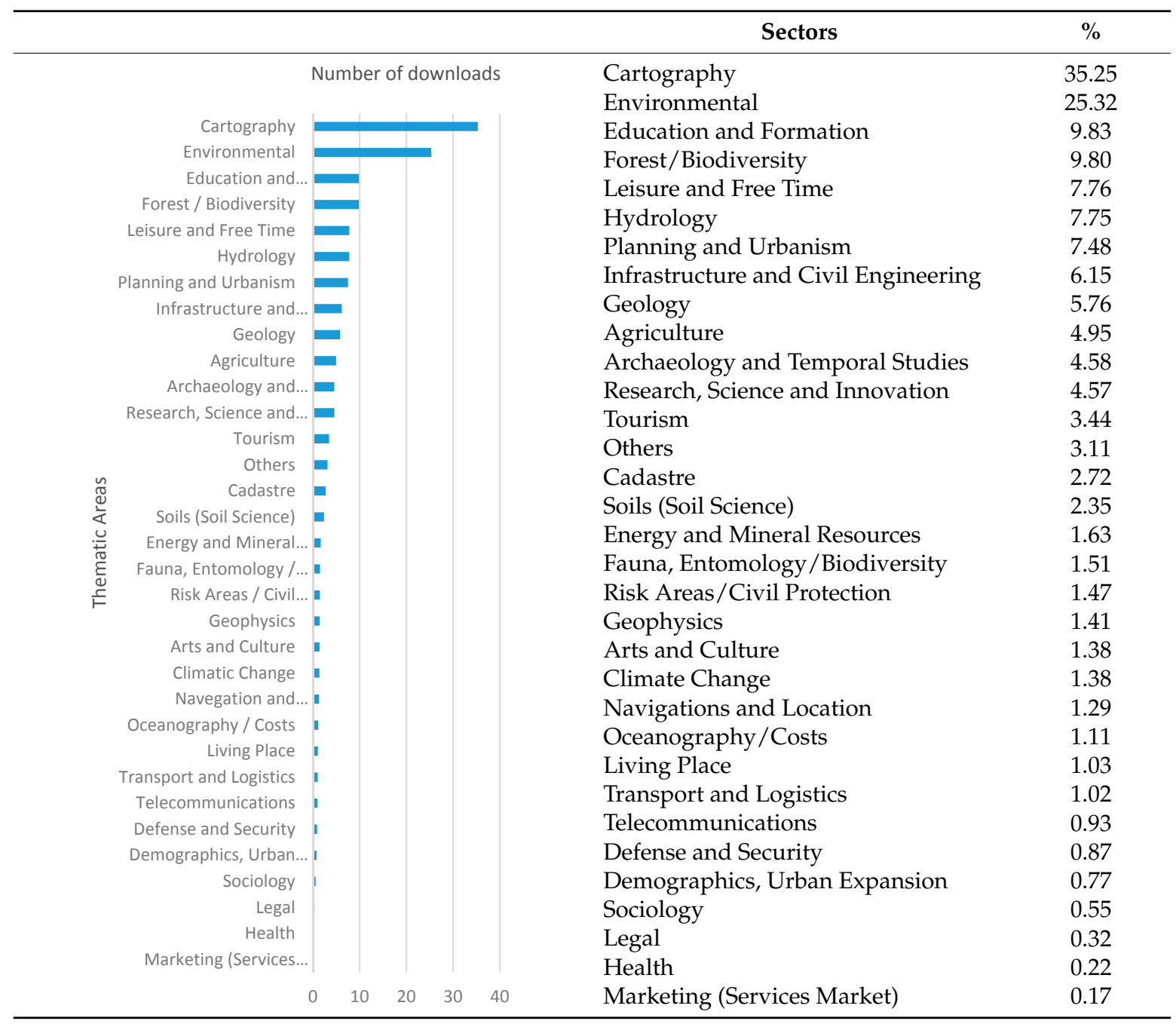

There is a free-text item for comments regarding the "Thematic Area". It was used 580 times by users to include their remarks. These remarks have been standardized according to the above criteria. Users do not answer this item 11,913 times, which accounts for $95.36 \%$ of cases. In the 580 cases with answers only 287 cases specify relevant information, and the remaining cases (293) include rubbish information (e.g., strange codes, absurd texts, etc.). Therefore, there is $50 \%$ of useless cases. In Figure 8 we can see the percentage of each normalized response relative to the total of 287 responses specified. As we can see, though less in number these standardized comments follow a distribution very similar to the comments of the standardized "Description of intended use". This result is logical because people use this free-text item in order to explain the same idea. Also, it is noteworthy that the category "Valuation, Suggestions and Thanks" takes a high percentage of cases. This category was not present in the case of the standard "Description of intended use". This category includes many cases of thanks-giving and positive remarks about the products. 


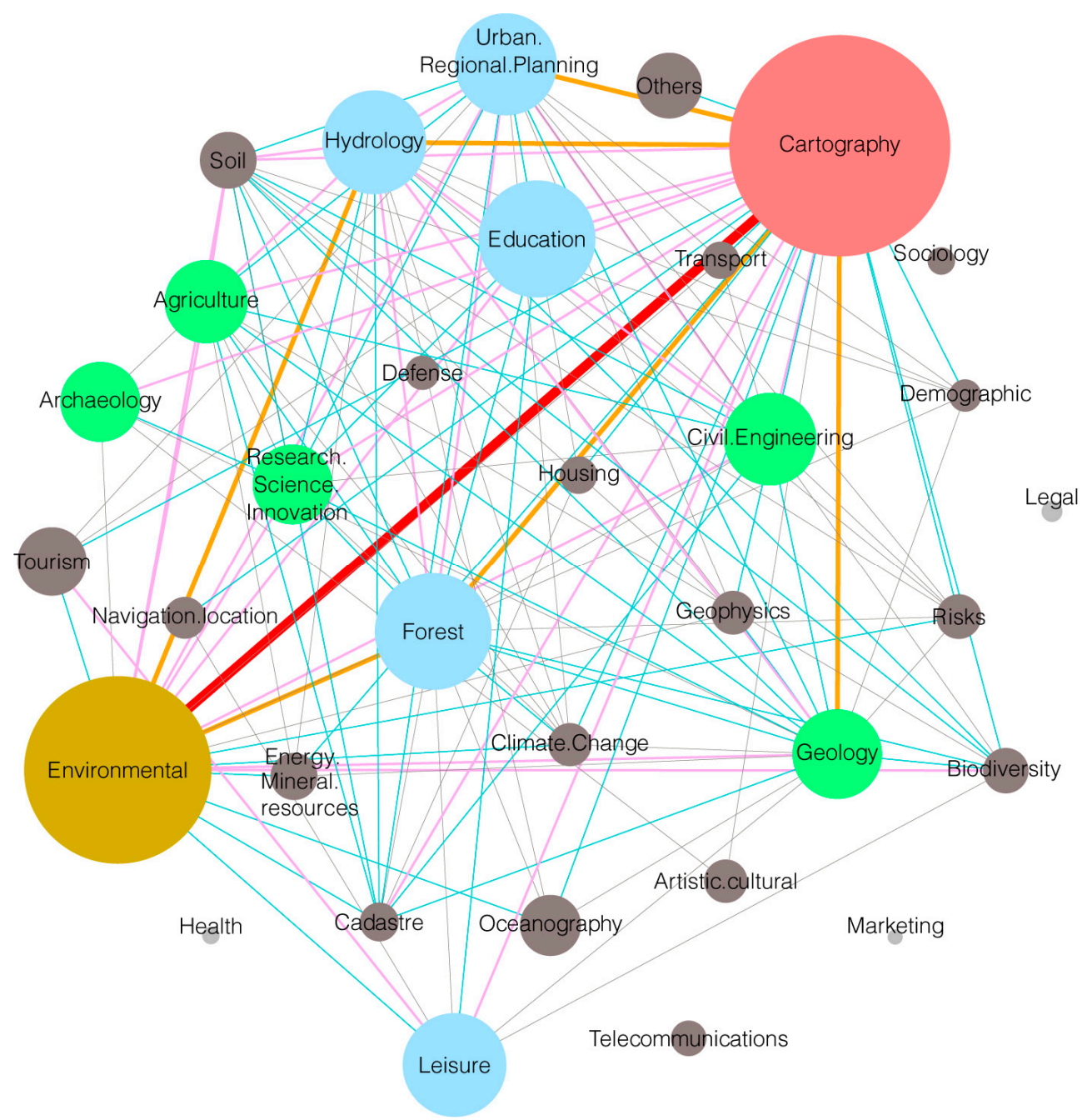

Figure 7. Thematic areas and their relations.
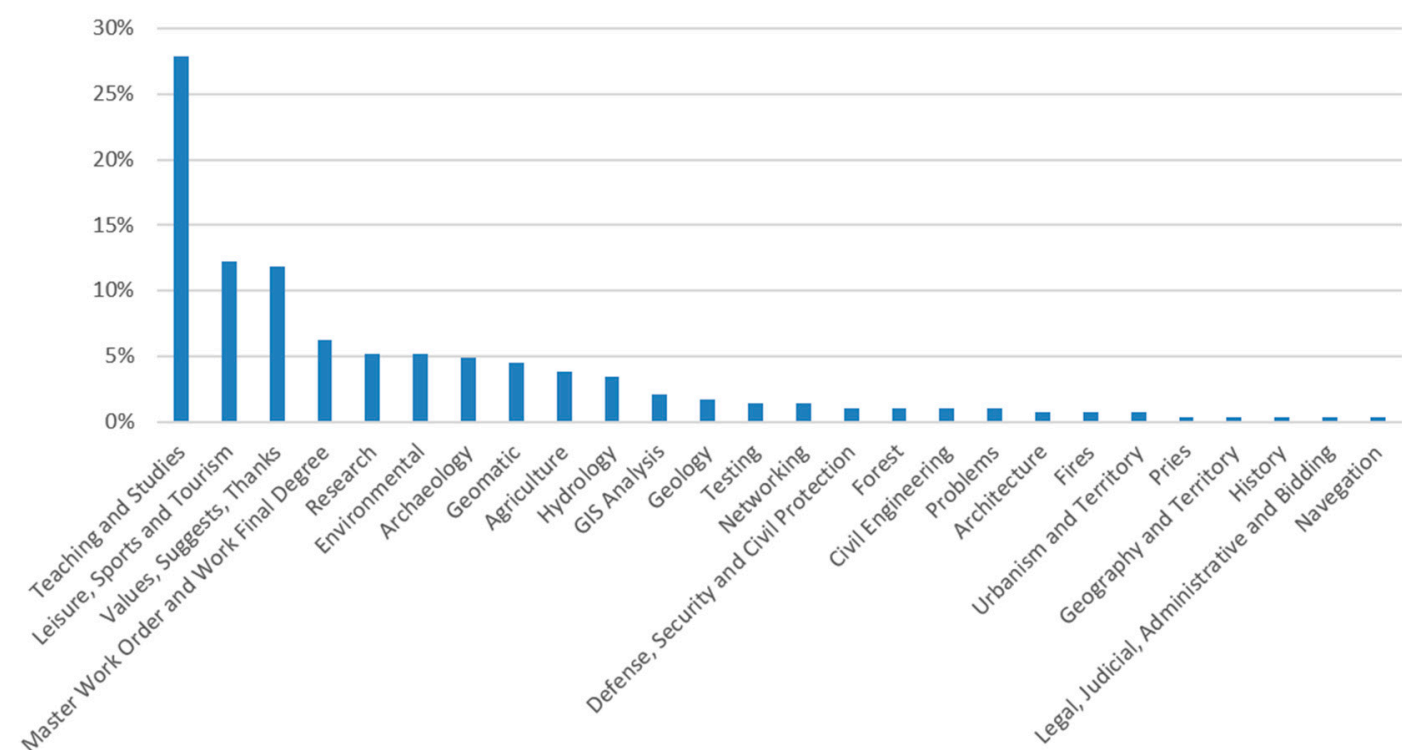

Figure 8. Percentage of responses normalized to total considered responses (287). 
If we establish a relationship between the selected Thematic Area and the type of DEM downloaded, we obtain Figure 9.

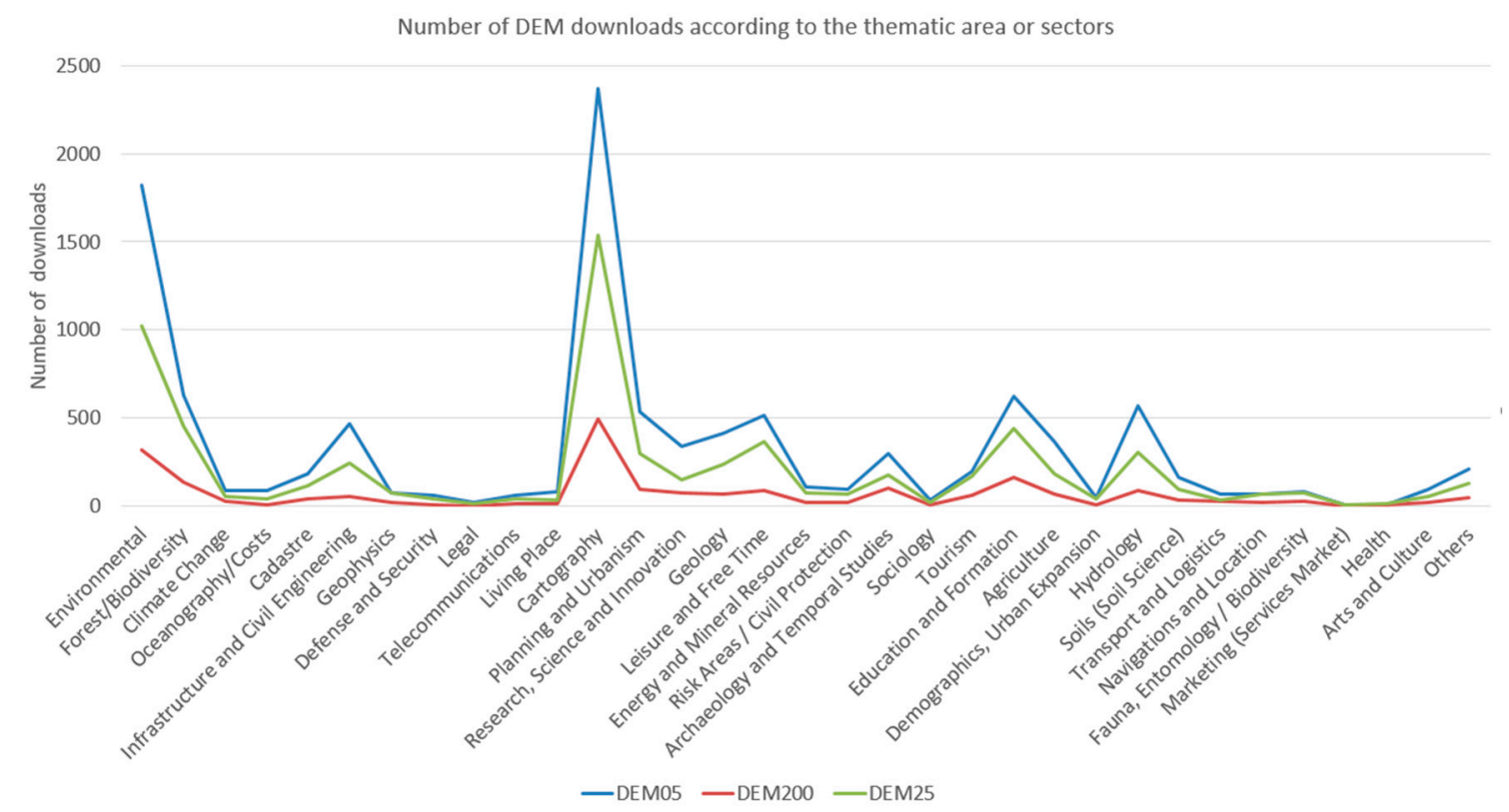

Figure 9. Number of Digital Elevation Model (DEM) downloads according to the Thematic Area.

In a first impression, the three curves follow a similar behavior in the different thematic areas. In those thematic areas that can work at any scale such as Cartography, Urbanism and Planning, Hydrology, Forestry and Biodiversity and the Environment, the curves keep an equidistance between the three types of DEM. Meanwhile in thematic areas such as Legal and Sociology the curves join, since in these studies we could not clearly define the scope of study and this is reflected in the similar downloads in the three types of DEM. We also observed that in thematic areas such as Geophysics, Energy and Mineral Resources, Risk Areas/Civil Protection, Demography and Urban Expansion and Tourism the downloads of DEM05 and DEM25 are almost identical, while the downloads of DEM200 are much smaller. This is related to the possibility of studying at large and medium scales of these thematic areas. Finally, we see how in the areas of Marketing and Health the curves are practically reversed. This is typical of studies in a large area of territory, where the DEMs do not have the need for a large resolution and it is not a priority in the objective pursued in those studies.

Finally, we will conduct an analysis of the standardized item "Description of intended use", and for this purpose we use the first 43 terms of Table 2. In this process only one term is assigned to each remark given by a user. Table 8 shows the standardized terms, the total account of cases and the corresponding percentage. We wish to emphasize that the survey requires users to complete this item, although the answers are not always useful. So there are more than $14 \%$ of cases with rubbish information (e.g., single letters, numbers, nonsense, insults, etc.), and these cases are labeled as Unspecified in Table 8. 
Table 8. Downloads of DEMs regarding the Description of use for which they are intended.

\begin{tabular}{|c|c|c|}
\hline Uses Standard Terms & Numbers of Records & Percentage of the Total \\
\hline Acoustic & 28 & 0.2 \\
\hline Agriculture & 190 & 1.5 \\
\hline GIS Analysis & 171 & 1.3 \\
\hline Archaeological Heritage & 271 & 2.2 \\
\hline Architecture & 29 & 0.2 \\
\hline Aviation & 12 & 0.1 \\
\hline Biology & 3 & 0.0 \\
\hline Cadastre Market & 103 & 0.8 \\
\hline Testing & 19 & 0.2 \\
\hline Curiosisty & 81 & 0.6 \\
\hline Security Defense Civil Protection & 81 & 0.6 \\
\hline Teaching and Studies & 3507 & 28.1 \\
\hline Renewable Energy & 51 & 0.4 \\
\hline Forest & 180 & 1.4 \\
\hline Geography Territory & 83 & 0.7 \\
\hline Geology & 188 & 1.5 \\
\hline Geomatic & 926 & 7.4 \\
\hline Hydrology & 515 & 4.1 \\
\hline History & 62 & 0.5 \\
\hline Environmental Impact & 37 & 0.3 \\
\hline Fires & 35 & 0.2 \\
\hline Civil Engineering & 184 & 1.5 \\
\hline Research & 723 & 5.8 \\
\hline Legal Judicial Administrative Tender & 25 & 0.2 \\
\hline Location & 27 & 0.2 \\
\hline Environment & 347 & 2.8 \\
\hline Mining & 20 & 0.2 \\
\hline Modeling & 31 & 0.2 \\
\hline Navegation & 39 & 0.3 \\
\hline Leisure Sports Tourism & 1189 & 9.5 \\
\hline Landscape & 40 & 0.3 \\
\hline Planning & 9 & 0.1 \\
\hline Problems & 2 & 0.0 \\
\hline Testing Data System & 116 & 0.9 \\
\hline Publications Disclosures & 63 & 0.5 \\
\hline Networks & 46 & 0.4 \\
\hline Natural Hazard & 22 & 0.2 \\
\hline Unspecified & 1762 & 14.1 \\
\hline Telecommunications & 31 & 0.2 \\
\hline Master Work Order and Work Final Degree & 839 & 6.7 \\
\hline Urbanism Territory & 194 & 1.5 \\
\hline Visualization & 44 & 0.4 \\
\hline 3D Visualization & 120 & 1.0 \\
\hline
\end{tabular}

The bold terms of Table 8 are those with a frequency greater than $5 \%$, here the "Unspecified" case has been excluded. "Teaching and Studies" is the case described most frequently. For their specificity, the cases of "Master's degree final project and Bachelor's degree final project" have been considered separately. Together they reached $34.8 \%$, which is more than a third of the intended uses related to education. This ensures a great future potential with users of a high technical level. Also, this is in line with the profiles we have described above.

The term "Leisure Sports and Tourism" reaches almost 10\% of the total and is set as the second term most used by users. It is interesting to observe how leisure activities are so important among users of DEMs. This suggests the existence of business opportunities by creating value-added products focused on specific sports. Under the term "Geomatics" we have included all the mapping activities (Geodesy, Cartography, Topography, Photogrammetry, etc.), so a high percentage is reached given 
the high functionality of DEMs in this field. We believe that this is a primarily professional use, which includes freelances and small businesses.

Research is the last term that accounts for more than $5 \%$ of cases. This activity is closely related to other terms (Teaching and Studies and Master's and Bachelor's Degree Final Projects), which often include a research component. However, we considered it independently because it has been explicitly indicated by many users, and also for the remarkable technological implications of the research. Therefore, in the future we would expect the existence of highly trained professionals and many scientific and educational publications based on DEMs.

Once we have described the terms of higher percentages, there is a diverse group of professional applications of great interest and importance. Within this group we can highlight the Hydrological applications (accounting for $4.1 \%$ of cases), and the applications related to the Environment, Archaeological Heritage, Agriculture, etc. All of these applications could be grouped under the heading of professional applications, which should also include Geomatics, and they would provide the importance of professional uses, in this case reaching more than $28 \%$ of cases.

In view of the above, we can group applications into three main types: first, applications for teaching and research uses; second, professional applications; and finally, sport and leisure uses. There are other answers that have not been discussed so far which we also consider it important to mention. In particular, there are those that have been normalized with the following terms: "Problems", "Curiosity" and "3D Visualization". Some comments indicate problems with the data, mainly with downloads and formats, but they are scarce. Although it is not the most suitable site for these comments, some users use this field for that purpose. The normalized term "Curiosity" provides entries that demonstrate an attitude of exploration of the product by the user; the user seeks a first contact with the product in order to discover its functionality. Finally, the normalized term "3D Visualization" includes entries related to the development of visual and immersive environments, and we would like to highlight these cases for their presumed importance for the future.

If we relate the standard comments of use with the type of DEM downloaded, we obtain Figure 10.

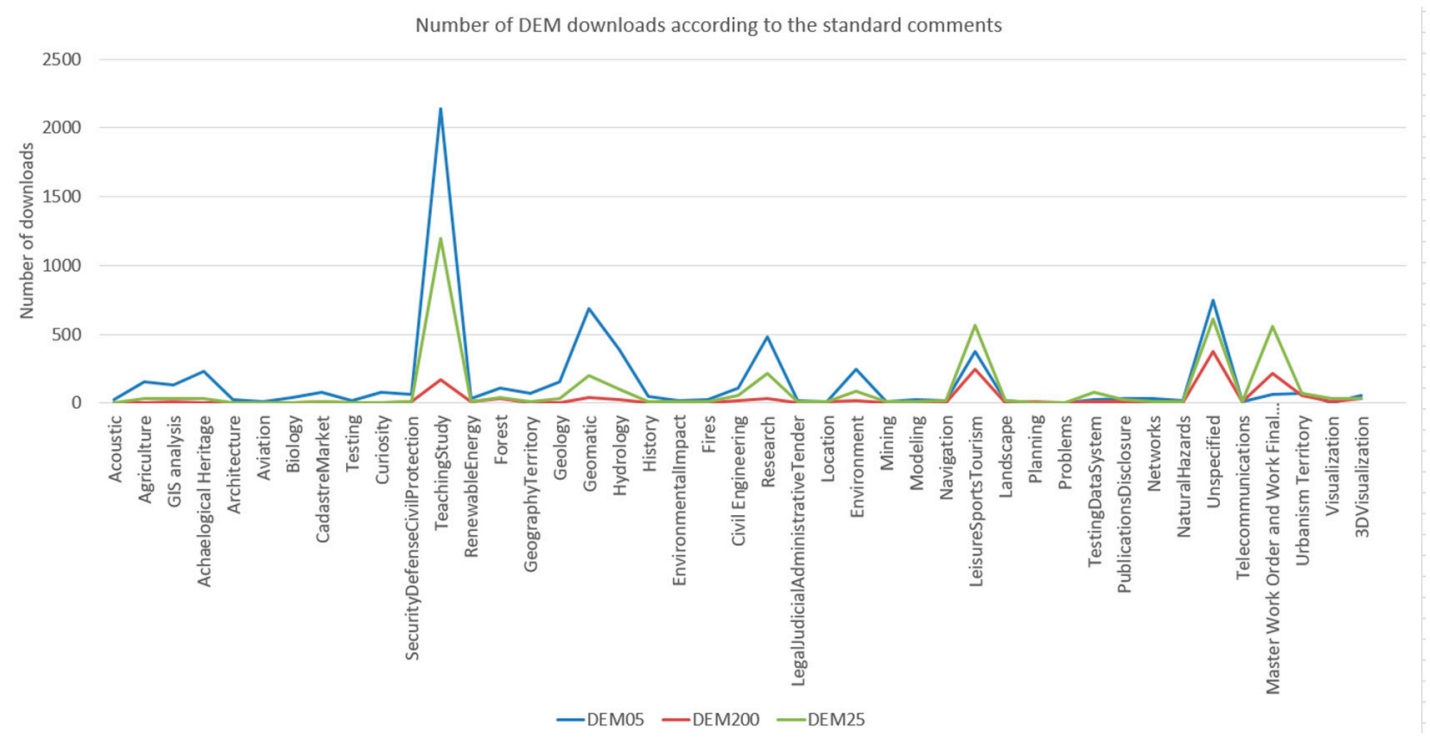

Figure 10. Number of DEM downloads according to the standard comments.

We observe how the highest percentages of downloads of digital terrain models are of higher resolution (DEM05) in almost all areas, so this occurs in: Agriculture, GIS analysis, archeology, architecture, Teaching and Study, Geology, Hydrology, Environmental Impact, Civil Engineering, Research, Environment, etc. As happened with the thematic areas, it seems logical that in certain studies the use of high resolution is more frequent to cover a limited extent, however models with 
lower resolution can be used for larger extensions, although it is usually less frequent. In "Leisure Tourism and Sports", in "Testing Data System" and in the "Master Work Order and Work Final Degree", the most downloaded DEM is that of intermediate resolution. This may be logical since for sports activities it is not necessary to have the highest resolution of the DEM. In the case of "Testing Data System" and "Master Work Order and Work Final Degree" there is no reasonable explanation to justify this type of resolution and no other. The use of DEM05 would be more justified in the master's and final degree projects, since in the use of "Teaching and Study" it is like that. Also in the thematic areas DEM05 is the most frequent in the field of research and education and training.

The fact that more intermediate resolution models are used in "Master Work Order and Work Final Degree", we think is because the user who has commented on this use is a university user who is heading towards the completion of their work and they are generally studies of medium territorial extension and mainly related to environmental issues.

\subsection{Valuation of the Product}

The valuation of the product by users is the last aspect to be analyzed in this study. To this end the survey provides two items, "Utility" and "Need to update the product". These items are valued numerically, and there are also two free text items for the user to make comments. First we will analyze the utility and then the need to update.

Figure 11 presents the frequencies of the assessment of the level of utility that users have assigned to each product (DEM05, DEM25 and DEM200). Higher values mean higher utility (see Table 1 for possible values). Users mark mostly the highest level of utility for all three products. If you look at the DEM of highest spatial resolution (DEM05) the utility value 5 scores more than twice the utility value 4 , and a third of users consider values between 4 and 3. The same thing happens with DEM200 and DEM25.

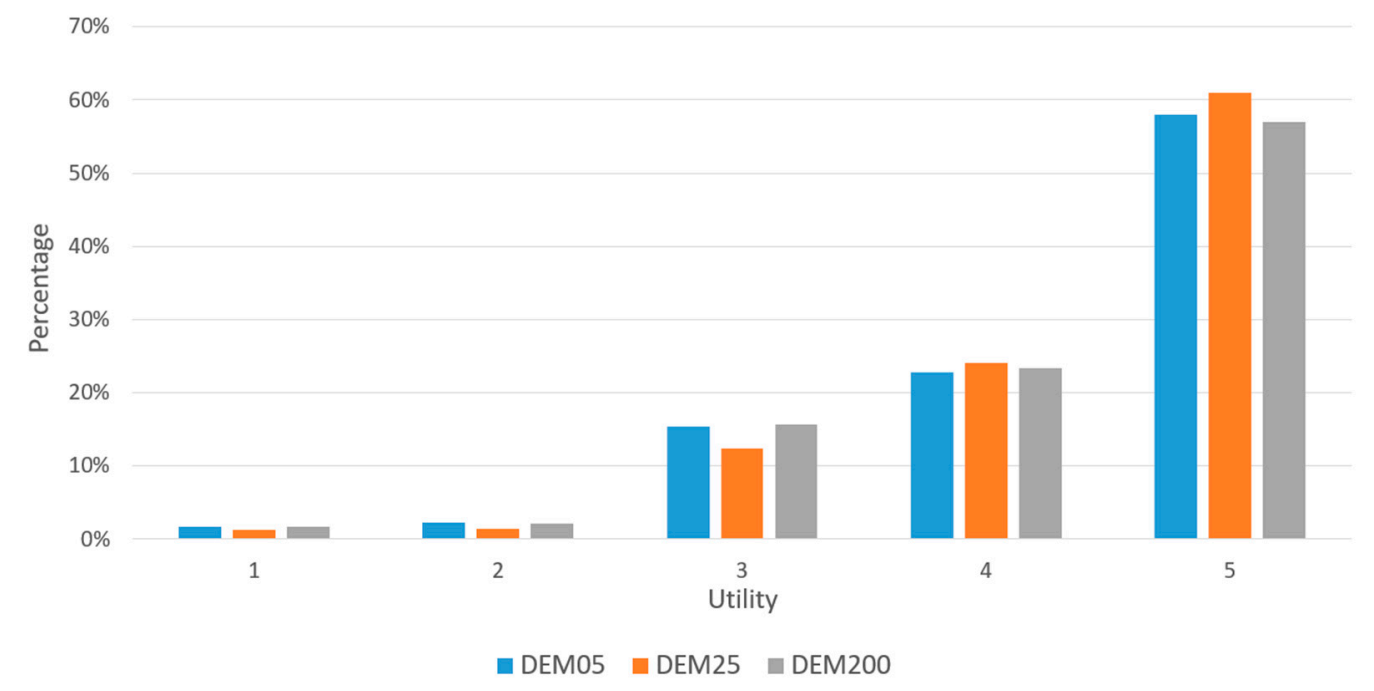

Figure 11. Percentage of utility levels in each type of DEM.

Considering all products together, Table 9 shows the distribution of valuations. The percentage of cases that consider the utility value 5 reaches $58.9 \%$. It is remarkable that the percentages for the utility values 1 and 2 are very low, not reaching $4 \%$ together. It is well known that in many surveys that apply Likert's scale users avoid selecting extreme values; however, we believe that our case is not the same. If the survey respondent knows the possibilities of DEMs, it would be illogical to think that he is going to respond to this item with a low value. So why are there values of 1 and 2 ? We believe that these correspond to atypical responses, that is, users are assessing other aspects or are simply mistaken when answering. In conclusion, we can say that the DEMs are generally considered very useful. 
Table 9. Utility levels as users of DEM.

\begin{tabular}{cc}
\hline Utility Level & Percentages Download (\%) \\
\hline 1 & 1.6 \\
2 & 1.9 \\
3 & 14.4 \\
4 & 23.2 \\
5 & 58.9 \\
\hline
\end{tabular}

The other issue discussed in this section is the assessment of "Need to update". It is not a direct evaluation of the product, but guides us regarding a trait that these products must have. Figure 12 shows the relative frequency histogram for each product and for each option of the assessment. Once again, the three products (DEM05, DEM25 and DEM200) are valuated in a very similar way, with small differences in percentage values. But Figure 7 shows a behavior very different from that of Figure 11. The need to update is a minor item for most users. In this case value 5 is not so far from value 4 . Value 3 reaches $25 \%$ and values 1 and 2 cannot be considered as outlier answers.

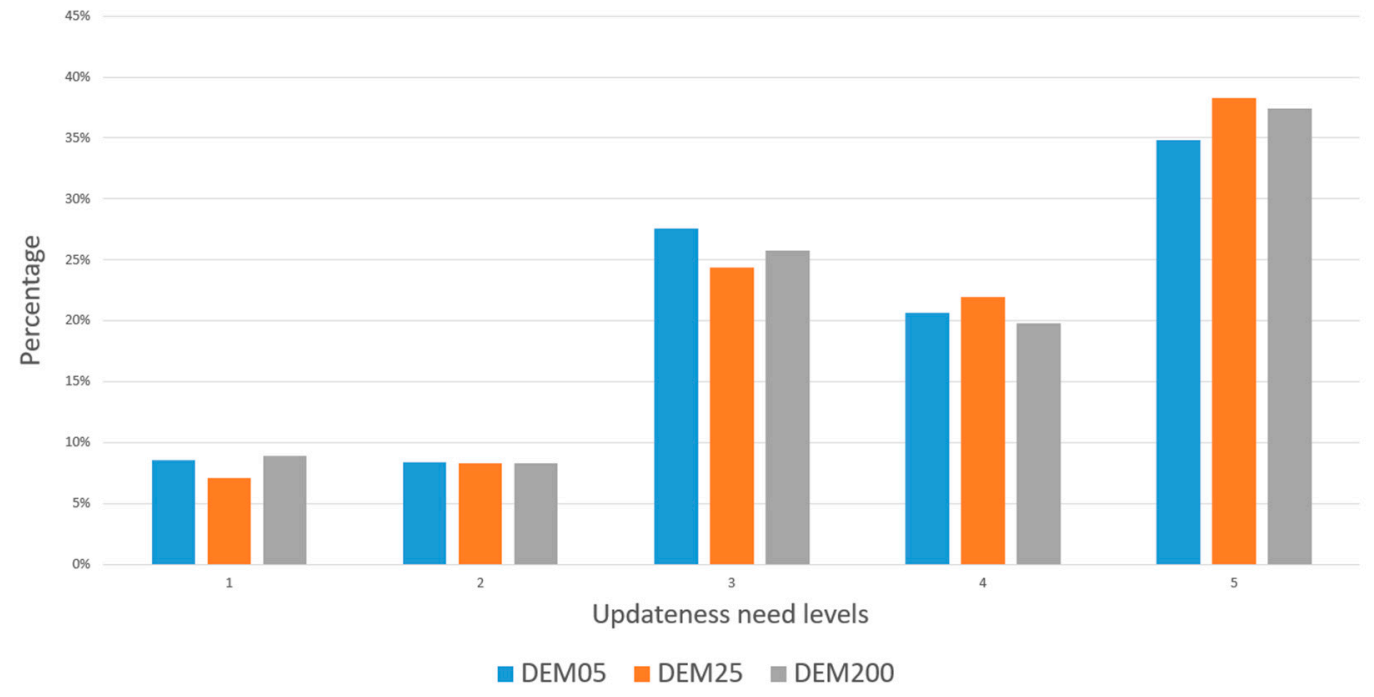

Figure 12. Percentage of Need to Update levels for each type of DEM.

Table 10 presents the aggregate data for the three products. As indicated above, in this case the percentages are somewhat more divided than in the assessment of utility. Thus, the percentage of value 5 is $36.2 \%$ and the lowest values ( 1 and 2 ) accumulate $16.5 \%$, a remarkable amount. In addition, we note that the sequence of values from level 5 up to 3 do not follow a trend of gradual decline (level 3 exceeds level 4), as in the utility-assessment case. In this case we believe that the frequency peak that occurs at level 3 is due to the bias to the central value that may appear in the answers to questions when using Likert's scale [17].

Table 10. Need to Update levels according to users of DEMs.

\begin{tabular}{cc}
\hline Need to Update Level & $\%$ \\
\hline 1 & 8.1 \\
2 & 8.4 \\
3 & 26.3 \\
4 & 21.0 \\
5 & 36.2 \\
\hline
\end{tabular}


The cross tabulation absolute frequencies between Need to Update and Utility scores are shown in Table 11. A Pearson Chi squared test indicates that there is no statistical independence between both variables, which implies that both variables have a great correlation. This situation is shown by Figure 13 where it can be observed, in normalized axis, that the points representing the same values of Need to Update and Utility are close to each other.

Table 11. Correspondence of cases between Updateness-need and Utility scores.

\begin{tabular}{ccccccc}
\hline \multirow{2}{*}{ Utility } & \multicolumn{7}{c}{ Updateness-Need } \\
\cline { 2 - 7 } & $\mathbf{1}$ & $\mathbf{2}$ & $\mathbf{3}$ & $\mathbf{4}$ & $\mathbf{5}$ & Total \\
\hline 1 & 117 & 14 & 14 & 2 & 14 & 161 \\
2 & 29 & 109 & 34 & 14 & 8 & 194 \\
3 & 80 & 136 & 1042 & 121 & 56 & 1435 \\
4 & 90 & 228 & 609 & 1188 & 178 & 2293 \\
5 & 481 & 334 & 889 & 735 & 3299 & 5738 \\
Total & 797 & 821 & 2588 & 2060 & 3555 & 9821 \\
\hline
\end{tabular}

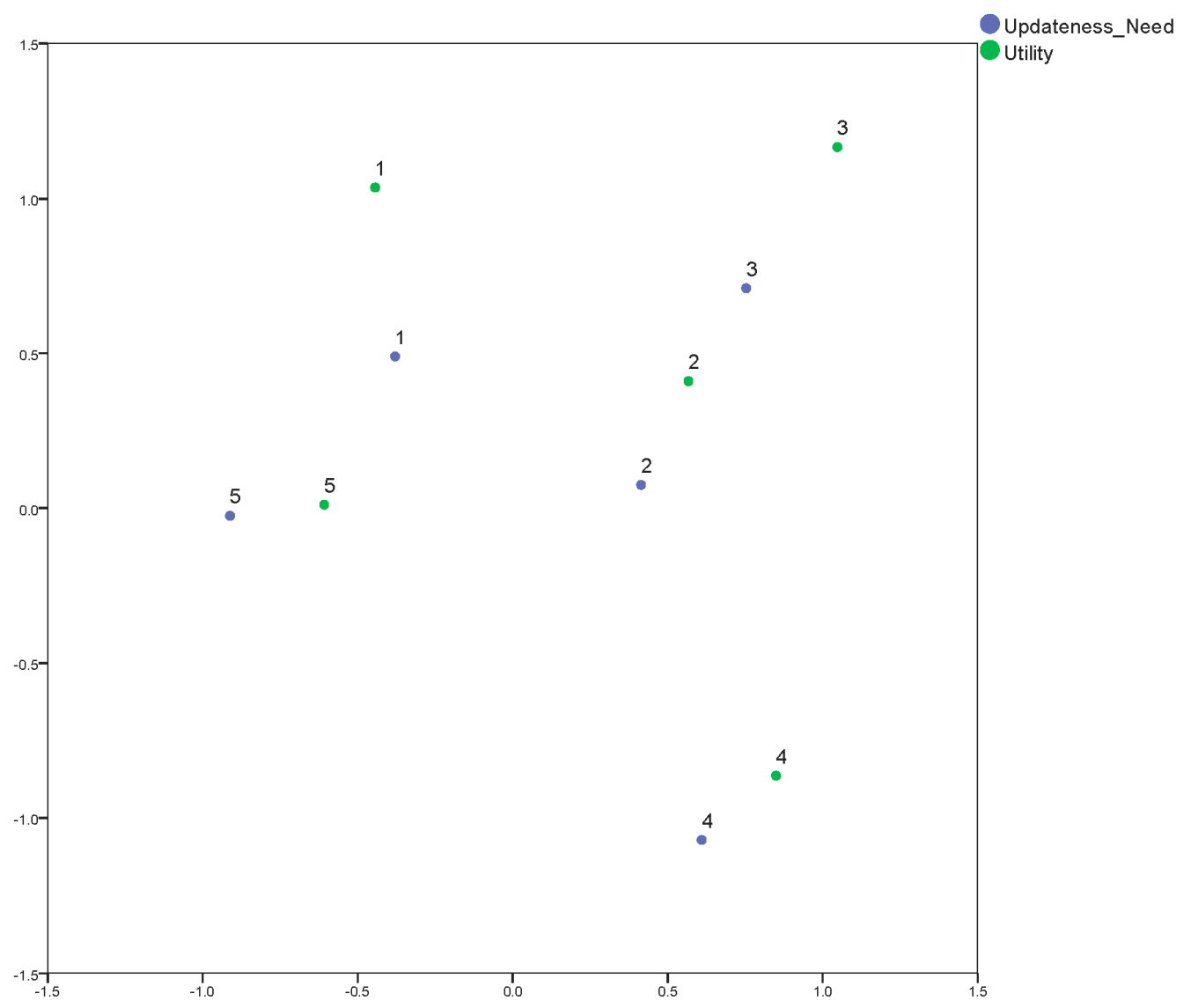

Figure 13. A Pearson Chi squared test between Utility and Need to Update.

There is a dependence between both variables because it is logical, since the user that considers the DEM very useful should consider that updating it is very necessary as well. This leads us to ponder whether it is necessary to include the two items in the survey, as maybe a single item that groups both ideas would have been sufficient. 


\section{Conclusions}

In this study, we have performed a characterization of users and uses of the DEMs that are downloaded on online services. For this purpose, we have used the download service of the Spanish cartographic agency (CNIG). We used a set of 12,493 records coming from a mandatory online survey developed at the time of downloading and for the year 2014. These surveys were provided by the CNIG. These surveys refer to the three DEM products available in the CNIG (DEM05, DEM25 and DEM200). In view of the data and its analysis we can conclude the following regarding the following aspects:

- Location. As would be expected, the country with the highest number of downloads is Spain, nevertheless there are some downloads in other countries, most often in neighboring countries (France and Portugal). Within Spain, the total amount of discharges is directly related to the province's total population. When discharges are analyzed in relation to the population of each province (as per thousand), the situation differs very much from the previous one and the relation is not so clear. The presence of regional mapping agencies, with DEM download services, affects our data in a national context.

- Downloaded Product. DEM05 is the most downloaded product, and this is the DEM of highest spatial resolution. We believe this is due to the fact that all users always want to access the data of highest possible resolution, whether or not it is strictly necessary.

- User profile. The predominant user profiles are: private users $(71 \%)$ and those related to universities (18\%). 60\% of users perform only one download through the period analyzed.

- Use of the product. The predominant uses are: (1) those related to teaching and research tasks of all types (universities, institutes, research centers, schools, various courses, etc.), accounting for $41 \%$ of cases; (2) uses of a professional nature (e.g., geomatics, hydrology, civil engineering, etc.) reaching 28\%, and finally; (3) uses related to leisure, sport and tourism, reaching 9.5\% of all cases.

- Valuation of the product. The assessment of the utility of the products is mainly $(60 \%)$ the highest value of the scale of the survey. The need to update the product is not considered as high as its utility. This situation seems perfectly logical, since the fundamental concern of data users is data existence. Currently available DEMs are relatively updated, so considering the temporal aspect is not entirely critical.

We understand that this study would not have been possible without the data from the surveys that were answered online, and for this reason we believe that we can also draw some conclusions about:

- Utility. The online survey is brief and focuses on essential aspects; its usefulness is great, as has been demonstrated in this paper. However, if the download systems of other mapping agencies are analyzed we can conclude that the use of such surveys is not widespread. We believe that, although the data are open, it is of great value to conduct a survey in order to understand the users and uses, and therefore propose improvements to products and services.

- Design. In relation to our data, we believe that the survey design is basic and functional but some improvements could be possible. For instance, the user registration system could include more information in order to allow a deeper analysis of the user's profiles. The items concerning "User Profile" and "Thematic Area" could provide a hierarchical structure of options so that users could choose their preferred option and allow easier analysis. The items that have greater problems are those that allow free text entry, however they provide very relevant information. In this case a system of natural language processing could facilitate this analysis.

Regarding our work, we think that one of the criticisms that can be made is that a partial perspective arises because we only focused on data from the online survey. The perspective of web services users or of those who have paid for the product have been left out of the analysis. It would be interesting to have data regarding these users to thereby allow a more complete analysis of users and uses of DEMs. Still, we believe that this analysis is a first step in this characterization and some conclusions of interest have been achieved in relation to users, uses and product assessment. 
Finally, we do not want to end without making a reflection on the interest in obtaining feedback from users. In the paradigm of SDI, based on the exploitation of standard, interoperable, open and free services, the user is essentially anonymous. Identifying the user, or requiring completion of a survey, breaks open service conditions. All these circumstances make difficult, and possibly biased, the results obtained from voluntary surveys. Very few download centers (linked to SDI at a national or regional level), have included voluntary surveys. But, as has been demonstrated with this analysis, an online survey is a very convenient tool for acquiring a better understanding of products, users and uses, which could be used for the improvement of products and services offered by the mapping agencies.

Acknowledgments: To Centro Nacional de Información Geográfica for offering us the data for this analysis. And also to users for completing the surveys.

Author Contributions: Mesa-Mingorance and Ariza-López conceived and designed the ideas to develop in the article and wrote it; Buenaño and Chicaiza performed the analysis of some data for obtaining figures and tables; Jianhong collaborated in the analysis of the data; Rodríguez-Pascual provided the data for the development of the study.

Conflicts of Interest: The authors declare no conflict of interest.

\section{References}

1. Real Decreto 1545/2007 de 23 de Noviembre, por el que se Regula el Sistema Cartográfico Nacional. Available online: http:/ / www.fomento.gob.es/Contraste/estilos.aspx?u=/mfom/lang_castellano/direcciones_generales / instituto_geografico/_informacion/normativa/norm_sect/sist_cart_nal/rd1545_2007.htm (accessed on 20 December 2017).

2. Decreto 141/2006, de 18 de Julio, por el que se Ordena la Actividad Cartográfica en la Comunidad Autónoma de Andalucía (BOJA N. 154, 09/08/2006). Available online: http:/ /www.juntadeandalucia.es/boja/2006/154/1 (accessed on 20 December 2017).

3. Yalcin, G. Initial Organizational Studies on National Spatial Data Infrastructure at Government Level. Procedia Technol. 2014, 12, 572-576. [CrossRef]

4. European Union. Directiva 2007/2/CE por la que se Establece una Infraestructura de Información Espacial en la Unión Europea (Inspire); European Directive 2007/2/CE which a Spatial Data Infrastructure is established in the European Union (Inspire); European Union: Brussels, Belgium, 2007.

5. European Commission (EC). The Socio-Economic Impact of the Spatial Data Infrastructure of Catalonia; European Commission Joint Research Centre: Luxembourg, 2008.

6. Juran, J.M.; De Feo Joseph, A. Juran's Quality Handbook, The Complete Guide to Performance Excellence, 6th ed.; ASQ: Milwaukee, WI, USA, 2010.

7. Ishikawa, K. What is Total Quality Control? The Japanese Way, 1st ed.; Prentice Hall: Upper Saddle River, NJ, USA, 1985.

8. EFQM. An Overview of the EFQM Excellence Model. 2013. Available online: http://www.efqm.org/sites / default/files/overview_efqm_2013_v1.pdf (accessed on 18 December 2017).

9. Deming, W.E. Out of the Crisis; MIT Center for Advanced Engineering Study: Cambridge, MA, USA, 1986.

10. IECA. NTCA 01-002. Modelo Aseguramiento Calidad de Productos de IG. Model Quality Assurance products Geographic Information. 2011. Available online: https://www.juntadeandalucia.es/ institutodeestadisticaycartografia/ieagen/sea/ntca/01_modelos/01002_Modelos_Aseguramiento_ Calidad.pdf (accessed on 18 December 2017).

11. Gomez, C.; Hayakawa, Y.; Obanawa, H. A study of Japanese landscapes using structure from motion derived DSMs and DEMs based on historical aerial photographs: New opportunities for vegetation monitoring and diachronic geomorphology. Geomorphology 2015, 242, 11-20. [CrossRef]

12. Saksena, S.; Merwade, V. Incorporating the effect of DEM resolution and accuracy for improved flood inundation mapping. J. Hydrol. 2015, 530, 180-194. [CrossRef]

13. Juel, A.; Groom, G.B.; Svenning, J.C.; Ejrnaes, R. Spatial Application of Random Forest models for fine-scale coastal vegetation classification using object based analysis of aerial orthophoto and DEM data. Int. J. Appl. Earth Obs. Geoinf. 2015, 42, 106-114. [CrossRef] 
14. Rekha, P.N.; Gangadharan, R.; Ravichandran, P.; Mahalakshmi, P.; Panigrahi, A.; Pillai, S.M. Assessment of impact of shrimp farming on coastal groundwater using Geographical Information System based Analytical Hierarchy Process. Aquaculture 2015, 448, 491-506. [CrossRef]

15. Stroeven, P.; Li, K.; Le, N.L.B.; He, H.; Stroeven, M. Capabilities for property assessment on diferent levels of the micro-strcutre of DEM-simulated cementitious materials. Constr. Build. Mater. 2015, 88, 105-117. [CrossRef]

16. Ley 14/2010 de 5 de julio, Sobre las Infraestructuras y los Servicios de Información Geográfica en España; (BOE $\mathrm{n}^{\circ}$ 163, 06/07/2010). Law 14/2010 of 5 of July on infrastructure and geographic information services in Spain; Boletín Oficial del Estado: Madrid, Spain, 2010.

17. Friedman, H.; Amoo, T. Rating the rating scales. J. Mark. Manag. 1999, 9, 114-123.

(C) 2017 by the authors. Licensee MDPI, Basel, Switzerland. This article is an open access article distributed under the terms and conditions of the Creative Commons Attribution (CC BY) license (http:/ / creativecommons.org/licenses/by/4.0/). 